\title{
Massive Connectivity with Massive MIMO-Part I: Device Activity Detection and Channel Estimation
}

\author{
Liang Liu, Member, IEEE and Wei Yu, Fellow, IEEE
}

\begin{abstract}
This two-part paper considers an uplink massive device communication scenario in which a large number of devices are connected to a base-station (BS), but user traffic is sporadic so that in any given coherence interval, only a subset of users are active. The objective is to quantify the cost of active user detection and channel estimation and to characterize the overall achievable rate of a grant-free twophase access scheme in which device activity detection and channel estimation are performed jointly using pilot sequences in the first phase and data is transmitted in the second phase. In order to accommodate a large number of simultaneously transmitting devices, this paper studies an asymptotic regime where the BS is equipped with a massive number of antennas. The main contributions of Part I of this paper are as follows. First, we note that as a consequence of having a large pool of potentially active devices but limited coherence time, the pilot sequences cannot all be orthogonal. However, despite the non-orthogonality, this paper shows that in the asymptotic massive multiple-input multiple-output (MIMO) regime, both the missed device detection and the false alarm probabilities for activity detection can always be made to go to zero by utilizing compressed sensing techniques that exploit sparsity in the user activity pattern. Part II of this paper further characterizes the achievable rates using the proposed scheme and quantifies the cost of using non-orthogonal pilot sequences for channel estimation in achievable rates.
\end{abstract}

Index Terms-Compressed sensing, approximate message passing (AMP), state evolution, massive connectivity, massive multiple-input multiple-output (MIMO), Internet-of-Things (IoT), machine-type communications (MTC).

\section{INTRODUCTION}

\section{A. Motivation}

Massive connectivity is a key requirement for future wireless cellular networks that aim to support Internet-ofThings (IoT) and machine-type communications (MTC). In a massive device connectivity scenario, a cellular basestation (BS) may be required to connect to a large number of devices (in the order $10^{4}$ to $10^{6}$ ), but a key characteristic of

Manuscript received June 17, 2017, revised November 9, 2017 and January 30, 2018, accepted March 8, 2018. The materials in this paper have been presented in part at the IEEE International Symposium on Information Theory (ISIT), Aachen, Germany, July 2017 [1]. The associate editor coordinating the review of this paper and approving it for publication was Dr. Mathini Sellathurai. This work is supported by Natural Sciences and Engineering Research Council (NSERC) of Canada via a discovery grant, a Steacie Memorial Fellowship and the Canada Research Chairs program.

The authors are with The Edward S. Rogers Sr. Department of Electrical and Computer Engineering, University of Toronto, Toronto, Ontario, Canada, M5S3G4, (e-mails:lianguot.liu@utoronto.ca; weiyu@comm.utoronto.ca). the IoT and MTC traffic is that the device activity patterns are typically sporadic so that at any given time only a small fraction of potential devices are active [2]. The sporadic traffic pattern may be due, for example, to the fact that often devices are designed to sleep most of the time in order to save energy and are activated only when triggered by external events, as typically the case in a sensor network. In these scenarios, the BS needs to dynamically identify the active users before data transmissions take place. The central question this paper seeks to address is how to quantify the cost of device activity detection and to account for its impact on the overall achievable rate in the cellular system design.

This paper considers the uplink of a single-cell massive connectivity scenario consisting of a BS equipped with $M$ antennas connected to a pool of $N$ potential devices, of which a fraction of $K$ devices are active at any given time, as shown in Fig. 11 In each coherence time interval $T$, the BS needs to identify the active devices, to estimate their channels and to decode the transmitted messages from the devices. In particular, a two-phase multiple-access scheme is adopted in which joint activity detection and channel estimation are performed using pilot sequences in the first phase of duration $L$, while data transmission takes place in the second phase of duration $T-L$, as shown in Fig. 2 . Note that we choose to combine user activity detection and channel estimation in order to minimize scheduling latency.

Observe that in order to achieve an appreciable data rate, $M$ has to be in the same order as $K$. This intuition can be justified from a degree-of-freedom (DoF) perspective by noting that when coherence time is large, the achievable DoF of a multiple-access system (without assuming a priori channel state information (CSI) at the receiver) is at maximal when the number of active devices equals to the number of receive antennas at the $\mathrm{BS}$ [3]. To accommodate a large number of active devices, the above result motivates us to consider the massive multiple-input multiple-output (MIMO) regime with large $M$.

Further, in order to achieve reasonably accurate uplink channel estimation, $L$ needs to be larger than $K$. Yet, since $L$ is constrained by the coherence time and the total number of potential devices $N$ can be very large, we usually have the situation where $N>L$. Thus, it is typically not possible to assign orthogonal pilot sequences to all the potential devices.

The main objectives of this paper are to quantify the performance of device activity detection and channel estimation when randomly generated non-orthogonal pilot sequences 


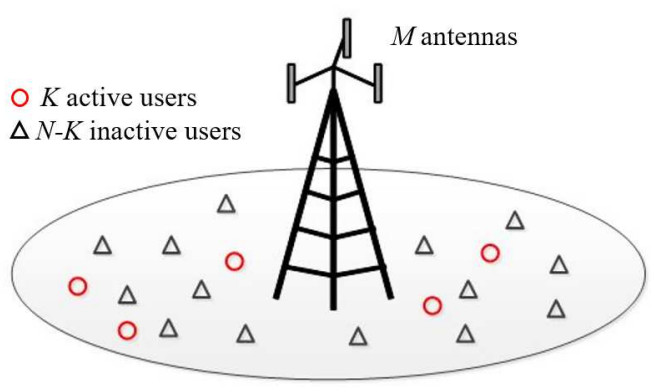

Fig. 1. System model of the massive device communication network.

\begin{tabular}{|c|c|}
\hline $\begin{array}{l}\text { User Detection and } \\
\text { Channel Training }\end{array}$ & Data Transmission \\
\hline $\begin{array}{l}T \\
L\end{array}$ & ${ }_{T-L}$ \\
\hline
\end{tabular}

Fig. 2. Two-phase transmission protocol.

are assigned for each device, and to examine its impact on the overall achievable data rate. Toward this end, we propose the use of approximate message passing (AMP) algorithm [4] in compressed sensing to exploit sparsity in device activity detection. We further analyze the resulting device detection performance and the channel estimation error by utilizing large-system result where $N, K, M$ and $L$ go to infinity in certain limits, thereby allowing a characterization of the overall achievable data rate for massive connectivity.

The main theoretical results of Part I of this paper show that massive MIMO is especially well suited for massive device connectivity in the sense that device activity detection error can always be driven to zero asymptotically in the limit as $M$ goes to infinity. Despite the asymptotically perfect detection, however, this paper also shows that the channel estimation error always remains-because of the use of nonorthogonal pilots - and that channel estimation error is the main cost of user activity detection on achievable rate. The analytical results of Part II of this paper [5] quantify this additional channel estimation error and shows its impact on achievable rate and optimal pilot length for massive connectivity.

\section{B. Prior Work}

Conventional cellular networks are designed based on the scheduling of active users to time or frequency slots. The overhead of scheduling massive number of sporadically active users over a separate control channel can, however, incur significant overhead. To this end, contention-based schemes have been proposed to deal with this issue. For example, [6]-[8] investigate a random access protocol in which each active user picks one of the orthogonal signature sequences at random and sends it to the BS, and a connection is established if the selected preamble is not used by the other users. The main drawback of this random access protocol is the need for contention resolution, since collision is unavoidable with a massive number of devices.

As an alternative, grant-free non-orthogonal user access schemes have recently attracted significant attention, where the active users send their pilot sequences to the BS simultaneously so that the BS can perform user activity detection, channel estimation, and/or data decoding in one shot [9]-[17]. In contrast to the traditional channel estimation process, the devices in these systems use non-orthogonal pilot sequences, due to the massive number of devices in the network and limited coherence time of the wireless channels. The key observation made in [9]-[17] is that the sparsity in user activity pattern allows the formulation of a compressed sensing problem. For example, assuming perfect CSI at the BS, a joint user activity and data detection for code division multiple access (CDMA) systems is investigated in [9], [10] by exploiting various sparsity structure. When CSI is not available at the BS, user activity detection and channel estimation is jointly performed in [11], [12], where [11] proposes a modified Bayesian compressed sensing algorithm in a cloud radio-access network, and [12] introduces a oneshot random access protocol and employs a basis pursuit denoising method with a detection error bound in an orthogonal frequency division multiplexing (OFDM) system. Moreover, [13]-[16] perform joint information decoding in addition based on various compressed sensing techniques.

One of the open issues in the above line of work is the lack of a rigorous performance analysis for the nonorthogonal multiple-access scheme for massive connectivity. Toward this end, the recent work of [17], [18] proposes the use of the AMP algorithm for the joint user activity detection and channel estimation problem, and further shows that a state evolution analysis [19] of the AMP algorithm allows an analytic characterization of the missed detection and false alarm probabilities for device detection. The analysis of [17], [18] is however quite involved, especially when the BS is equipped with multiple antennas. Further, [17], [18] analyze device detection performance only. Its impact on user achievable rate has not been investigated.

This paper makes further progress in this direction by making an observation that the characterization of device detection performance and user achievable rates can be simplified substantially in the massive MIMO regime where the number of BS antennas goes to infinity. In particular, our asymptotic analysis provides a key insight that the channel estimation error, rather than detection error, is the limiting factor in the massive MIMO regime.

We emphasize that while massive MIMO system has been extensively studied in the literature [20]-[24], conventional analysis focuses on the regime of small number of users as compared to the number of BS antennas. By contrast, the analysis of this paper assumes that the number of devices can also go to infinity, while focusing on the cost of device detection in the overall design. We also note here that the information theoretic upper bounds for massive connectivity 
have been derived in [3], [25] for both the cases with and without CSI, respectively. However, how to approach these rate upper bounds in a practical massive connectivity system is still an open problem.

The main tool in our analysis is the AMP algorithm, which is originally proposed to solve the single measurement vector (SMV) based sparse signal recovery problem [4], then generalized to the multiple measurement vector (MMV) problem in [26], [27], corresponding to the multiple-antenna case in our setting. The AMP algorithm is also generalized to the case where the input signal is not independently and identically distributed (i.i.d.) in [28]. As compared to other compressed sensing techniques, AMP has many appealing advantages. First, the AMP algorithm has low complexity similar to other iterative thresholding algorithms while achieving the reconstruction power of the basis pursuit at the same time [4]. Second, the performance of the AMP algorithm can be accurately predicated by state evolution [19].

\section{Main Contributions}

This two-part paper provides an analytical performance characterization of the two-phase transmission protocol in a single-cell massive connectivity scenario with massive MIMO, in which the active users send their non-orthogonal pilot sequences to the BS simultaneously for user activity detection and channel estimation in the first phase of the coherence time, then transmit data to the BS for information decoding in the second phase. By studying certain asymptotic regime where the number of BS antennas, the number of potential devices, and the number of active devices all go to infinity, this paper is able to analyze the performance of user activity detection and channel estimation, and further to characterize the overall achievable rates while taking the cost of both user activity detection and channel estimation into consideration. Specifically, the main contributions of this paper are as follows.

In Part I of this paper, we design a minimum meansquared error (MMSE) denoiser in the vector AMP algorithm for user activity detection and channel estimation based on statistical channel information. By exploiting the state evolution of vector AMP, we show that the MMSEbased AMP algorithm is capable of driving the user detection error probability down to zero as the number of antennas at the BS goes to infinity. This implies that perfect user activity detection is possible in a practical IoT/MTC system if the BS is equipped with a sufficiently large number of antennas. Further, the statistical distributions of the estimated channels can also be obtained analytically thanks to the state evolution. These results are used in Part II of this paper [5] to characterize achievable transmission rates of massive connectivity.

\section{Organization}

The rest of Part I of this paper is organized as follows. Section $\amalg$ describes the system model for massive connectiv- ity and introduces the two-phase transmission protocol for user detection, channel estimation, and data transmission. Section 피 presents the vector AMP based user activity detection and channel estimation scheme; Section IV analyzes its detection error probability and channel estimation error performance; Section $\mathrm{V}$ further provides an asymptotic analysis in the massive MIMO regime. Section VI provides the numerical simulation results pertaining to device detection and channel estimation error. Finally, Section VII concludes this Part I of the paper.

\section{E. Notation}

Scalars are denoted by lower-case letters, vectors by boldface lower-case letters, and matrices by bold-face uppercase letters. The identity matrix and the all-zero matrix of appropriate dimensions are denoted as $\boldsymbol{I}$ and $\mathbf{0}$, respectively. For a matrix $\boldsymbol{M}$ of arbitrary size, $\boldsymbol{M}^{H}$ and $\boldsymbol{M}^{T}$ denote its conjugate transpose and transpose, respectively. The expectation operator is denoted as $\mathbb{E}[\cdot]$. The distribution of a circularly symmetric complex Gaussian (CSCG) random vector with mean $\boldsymbol{x}$ and covariance matrix $\boldsymbol{\Sigma}$ is denoted by $\mathcal{C N}(\boldsymbol{x}, \boldsymbol{\Sigma})$; the space of complex matrices of size $m \times n$ is denoted as $\mathbb{C}^{m \times n}$.

\section{SySTEM MODEL}

Consider the uplink of a single-cell cellular network consisting of $N$ users, denoted by the set $\mathcal{N}=\{1, \cdots, N\}$. It is assumed that the BS is equipped with $M$ antennas, while each user is equipped with one antenna. The complex uplink channel vector from user $n$ to the BS is denoted by $\boldsymbol{h}_{n} \in \mathbb{C}^{M \times 1}, n=1, \cdots, N$. This paper adopts a blockfading model, in which all the channels follow independent quasi-static flat-fading within a block of coherence time, where $\boldsymbol{h}_{n}$ 's remain constant, but vary independently from block to block. Moreover, we model the channel vector $\boldsymbol{h}_{n}$ as $\boldsymbol{h}_{n}=\sqrt{\beta_{n}} \boldsymbol{g}_{n}, \forall n$, where $\boldsymbol{g}_{n} \sim \mathcal{C N}(\mathbf{0}, \boldsymbol{I})$ denotes the Rayleigh fading component, and $\beta_{n}$ denotes the path-loss and shadowing component. Therefore, we have $\boldsymbol{h}_{n} \sim \mathcal{C N}\left(\mathbf{0}, \beta_{n} \boldsymbol{I}_{n}\right), \forall n$. The path-loss and shadowing components depend on the user location and are assumed to be known at the BS, 1

The sporadic nature of user traffic is modeled as follows. We assume that the users are synchronized and each user decides in each coherence block whether or not to access the channel with probability $\epsilon$ in an i.i.d. manner. Thus, within each coherence block only a subset of the users are active. In each particular block, we define the user activity indicator for user $n$ as follows:

$$
\alpha_{n}= \begin{cases}1, & \text { if user } n \text { is active, } \quad \forall n, \\ 0, & \text { otherwise }\end{cases}
$$

\footnotetext{
${ }^{1}$ In this paper we mainly focus on the scenario where the devices are stationary, e.g., home security systems, smart metering systems, etc.
} 
so that $\operatorname{Pr}\left(\alpha_{n}=1\right)=\epsilon, \operatorname{Pr}\left(\alpha_{n}=0\right)=1-\epsilon, \forall n$. Further, we define the set of active users within a coherence block as

$$
\mathcal{K}=\left\{n: \alpha_{n}=1, n=1, \cdots, N\right\} .
$$

We denote the number of active users as $K$, i.e., $K=|\mathcal{K}|$. The overall channel input-output relationship is modeled as:

$$
\boldsymbol{y}=\sum_{n} \boldsymbol{h}_{n} \alpha_{n} x_{n}+\boldsymbol{z}=\sum_{k \in \mathcal{K}} \boldsymbol{h}_{k} x_{k}+\boldsymbol{z},
$$

where $x_{n} \in \mathbb{C}, \boldsymbol{y} \in \mathbb{C}^{M \times 1}$, and $\boldsymbol{z} \in \mathbb{C}^{M \times 1}$ respectively are the user transmitted signal, the channel output at the BS, and the additive white Gaussian noise (AWGN) vector distributed as $\mathcal{C N}\left(\mathbf{0}, \sigma^{2} \boldsymbol{I}\right)$. For simplicity, this paper assumes no power adaptation so that all the devices transmit at a constant power $\rho$, i.e.,

$$
\mathbb{E}\left|x_{n}\right|^{2}=\rho .
$$

Further, we do not account for intercell interference. The objective at the BS is to detect which users are active and to decode their transmitted messages within each coherence block.

This paper adopts a grant-free multiple-access scheme as shown in Fig. 2] in which each coherence block of length $T$ symbols is divided into two phases. In the first phase, the active users send their pilot sequences of length $L$ symbols to the BS synchronously, and the BS jointly detects the user activities, i.e., $\alpha_{n}$ 's, as well as the active users' channels, i.e., $\boldsymbol{h}_{n}$ 's, $\forall n \in \mathcal{K}$. In the second phase, the active users send their data to the BS using the remaining $T-L$ symbols, and the BS decodes these messages based on the knowledge of user activities and channels obtained in the first phase.

For the massive connectivity scenario with a large number of potential devices, the length of pilot sequence is typically smaller than the total number of devices, i.e., $L<N$. In this case, it is not possible to assign mutually orthogonal sequences to all the users. Following the pioneering works on AMP [4], [19], [26]-[28], this paper assumes that pilot sequences are generated randomly, i.e., each user $n$ is assigned a unique pilot sequence

$$
\boldsymbol{a}_{n}=\left[a_{n, 1}, \cdots, a_{n, L}\right]^{T} \in \mathbb{C}^{L \times 1},
$$

whose entries are generated from i.i.d. complex Gaussian distribution with zero mean and variance $1 / L$, i.e.,

$$
a_{n, l} \sim \mathcal{C N}\left(0, \frac{1}{L}\right)
$$

so that each pilot sequence has unit norm, i.e., $\left\|\boldsymbol{a}_{n}\right\|^{2}=1$, as $L \rightarrow \infty$. It is further assumed that the pilot sequences of all the users are known by the BS.

To facilitate analysis, this paper considers a certain asymptotic regime where $N \rightarrow \infty$, so that $K \rightarrow \epsilon N$, and the empirical distribution of $\beta_{1}, \cdots, \beta_{N}$ converges to a fixed distribution denoted by $p_{\beta}$.

\section{User ACTIVITy Detection AND ChANNEL ESTIMATION VIA AMP}

In this section, we show that the AMP algorithm can be used in the first phase for user activity detection and channel estimation by exploiting the sparsity in user activity.

\section{A. Device Activity Detection and Channel Estimation as a Compressed Sensing Problem}

Consider the first phase of massive device transmission in which each user sends its pilot sequence synchronously through the channel. Define $\rho^{\text {pilot }}$ as the identical transmit power of the active users in the first transmission phase. The transmit signal of user $n$ can be expressed as $\alpha_{n} \sqrt{\xi} \boldsymbol{a}_{n}$, where $\xi=L \rho^{\text {pilot }}$ denotes the total transmit energy of each active user in the first phase 2 The received signal at the BS is then

$$
\boldsymbol{Y}=\sqrt{\xi} \sum_{n \in \mathcal{N}} \alpha_{n} \boldsymbol{a}_{n} \boldsymbol{h}_{n}^{T}+\boldsymbol{Z},
$$

where $\boldsymbol{Y} \in \mathbb{C}^{L \times M}$ is the matrix of received signals across $M$ antennas over $L$ symbols, and $\boldsymbol{Z}=\left[\boldsymbol{z}_{1}, \cdots, \boldsymbol{z}_{M}\right]$ with $\boldsymbol{z}_{m} \sim \mathcal{C N}\left(\mathbf{0}, \sigma^{2} \boldsymbol{I}\right), \forall m$, is the independent AWGN at the BS. Now define

$$
\boldsymbol{A}=\left[\boldsymbol{a}_{1}, \cdots, \boldsymbol{a}_{N}\right] .
$$

Let $\boldsymbol{x}_{n}=\alpha_{n} \boldsymbol{h}_{n}$ and define

$$
\boldsymbol{X}=\left[\boldsymbol{x}_{1}, \cdots, \boldsymbol{x}_{N}\right]^{T} .
$$

Then, the training phase can be modeled as the following matrix equation

$$
\boldsymbol{Y}=\sqrt{\xi} \boldsymbol{A} \boldsymbol{X}+\boldsymbol{Z}
$$

where the rows of the matrix $\boldsymbol{X}$ follow a Bernoulli Gaussian distribution:

$$
p \boldsymbol{x}_{n}=(1-\epsilon) \delta_{0}+\epsilon p_{\boldsymbol{h}_{n}}, \quad \forall n .
$$

Here, $\delta_{0}$ denotes the point mass measure at zero, and $p_{\boldsymbol{h}_{n}}$ denotes the distribution of user $n$ 's channel $\boldsymbol{h}_{n} \sim$ $\mathcal{C N}\left(\mathbf{0}, \beta_{n} \boldsymbol{I}\right)$.

The goal for the BS in the first phase is to detect the user activities and to estimate the user channels by recovering $\boldsymbol{X}$ based on the noisy observation $\boldsymbol{Y}$. As $\boldsymbol{X}$ is row sparse, i.e., many $\boldsymbol{x}_{n}$ 's are zero, such a reconstruction problem is a compressed sensing problem. Further, as the sparsity pattern is sensed at multiple antennas, this is an MMV compressed sensing setup.

Among many powerful compressed sensing techniques, this paper adopts a low-complexity AMP algorithm to recover the row-sparse matrix $\boldsymbol{X}$. Before proceeding to

\footnotetext{
${ }^{2}$ In practice, the pilot sequence length $L$ is large but finite. It is thus possible that the power of the randomly generated pilot $\sqrt{\xi} \boldsymbol{a}_{n}$ is slightly larger than $\xi$ for some users. To satisfy the transmit power constraint, in practice we can generate the pilot sequences based on a modified distribution: $a_{n, l} \sim \mathcal{C N}(0,1 / L-\zeta)$. With a careful choice of $\zeta$, the probability that one pilot sequence violates the power constraint can be close to zero.
} 
evaluate its performance for user activity detection and channel estimation, we first briefly review the vector version of the AMP algorithm in next subsection.

\section{B. Vector AMP Algorithm}

The vector AMP algorithm is first proposed in [26]. This paper considers a version of the algorithm proposed in [18 that aims to provide an estimate $\hat{\boldsymbol{X}}(\boldsymbol{Y})$ based on $\boldsymbol{Y}$ that minimizes the mean-squared error (MSE)

$$
\mathrm{MSE}=\mathbb{E}_{\boldsymbol{X} \boldsymbol{Y}}\|\hat{\boldsymbol{X}}(\boldsymbol{Y})-\boldsymbol{X}\|_{2}^{2}
$$

assuming the prior distribution (11) and the channel model [10). The algorithm is based on an approximation of the message passing algorithm for solving the above problem.

Starting with $\boldsymbol{X}^{0}=\mathbf{0}$ and $\boldsymbol{R}^{0}=\boldsymbol{Y}$, the general form of the vector AMP algorithm proceeds at each iteration as [18], [26], [28]:

$$
\begin{aligned}
\boldsymbol{x}_{n}^{t+1} & =\eta_{t, n}\left(\left(\boldsymbol{R}^{t}\right)^{H} \boldsymbol{a}_{n}+\boldsymbol{x}_{n}^{t}\right), \\
\boldsymbol{R}^{t+1} & =\boldsymbol{Y}-\boldsymbol{A} \boldsymbol{X}^{t+1}+\frac{N}{L} \boldsymbol{R}^{t} \sum_{n=1}^{N} \frac{\eta_{t, n}^{\prime}\left(\left(\boldsymbol{R}^{t}\right)^{H} \boldsymbol{a}_{n}+\boldsymbol{x}_{n}^{t}\right)}{N},
\end{aligned}
$$

where $t=0,1, \cdots$ is the index of the iteration, $\boldsymbol{X}^{t}=$ $\left[\boldsymbol{x}_{1}^{t}, \cdots, \boldsymbol{x}_{N}^{t}\right]^{T}$ is the estimate of $\boldsymbol{X}$ at iteration $t$, and $\boldsymbol{R}^{t}=\left[\boldsymbol{r}_{1}^{t}, \cdots, \boldsymbol{r}_{L}^{t}\right]^{T} \in \mathbb{C}^{L \times M}$ denotes the corresponding residual. Intuitively, the algorithm performs in (13) a matching filtering of the residual for each user $n$ using its pilot sequence, followed by a denoising step using an appropriately designed denoiser $\eta_{t, n}(\cdot): \mathbb{C}^{M \times 1} \rightarrow \mathbb{C}^{M \times 1}$. The residual is then updated in (14), but corrected with a so-called Onsager term involving $\eta_{t, n}^{\prime}(\cdot)$, the first-order derivative of $\eta_{t, n}(\cdot)$.

A remarkable property of the AMP algorithm is that when applied to the compressed sensing problem with the entries of the sensing matrix $\boldsymbol{A}$ generated from i.i.d. Gaussian distribution, its detection performance in certain asymptotic regime can be accurately predicted by the so-called state evolution. The asymptotic regime is when $L, K, N \rightarrow \infty$, while their ratios converge to some fixed positive values $N / L \rightarrow \omega$ and $K / N \rightarrow \epsilon$ with $\omega, \epsilon \in(0, \infty)$, while keeping the total transmit power fixed at $\xi$. Note that we fix the total transmit power rather than allowing it to scale with $L$ here in this hypothetical asymptotic system in order to carry out the state evolution analysis. (This implies that the per-symbol power goes down to zero in this hypothetical asymptotic regime.) The analysis is then used to predict the system performance at finite (but large) $L, K, N$ and $\xi=L \rho^{\text {pilot }}$. As shown in [17], this approach is found to corroborate very well with simulation results.

Specifically, given $\beta \sim p_{\beta}$, define a random vector $\boldsymbol{X}_{\beta} \in \mathbb{C}^{M \times 1}$ with a distribution $(1-\epsilon) \delta_{0}+\epsilon p_{\boldsymbol{h}_{\beta}}$, where $p_{\boldsymbol{h}_{\beta}}$ denotes the distribution $\boldsymbol{h}_{\beta} \sim \mathcal{C N}(\mathbf{0}, \beta \boldsymbol{I})$. Let $\boldsymbol{V} \in$ $\mathbb{C}^{M \times 1} \sim \mathcal{C N}(\mathbf{0}, \boldsymbol{I})$ be independent of $\boldsymbol{X}_{\beta}$. Then, the state evolution is the following recursion for $t \geq 0$ [18], [19], [26], [28]:

$$
\begin{aligned}
\boldsymbol{\Sigma}_{t+1}=\frac{\sigma^{2}}{\xi} \boldsymbol{I}+\omega \mathbb{E} & {\left[\left(\eta_{t, \beta}\left(\boldsymbol{X}_{\beta}+\boldsymbol{\Sigma}_{t}^{\frac{1}{2}} \boldsymbol{V}\right)-\boldsymbol{X}_{\beta}\right)\right.} \\
& \left.\left(\eta_{t, \beta}\left(\boldsymbol{X}_{\beta}+\boldsymbol{\Sigma}_{t}^{\frac{1}{2}} \boldsymbol{V}\right)-\boldsymbol{X}_{\beta}\right)^{H}\right],
\end{aligned}
$$

where $\boldsymbol{\Sigma}_{t}$ is referred to as the state, and the expectation is over $\beta, \boldsymbol{X}_{\beta}$ and $\boldsymbol{V}$. Note that in the above equation, $\eta_{t, n}(\cdot)$ is replaced by $\eta_{t, \beta}(\cdot)$ for convenience, since $n$ and $\beta_{n}$ are interchangeable. Moreover, the initial point to the above state evolution is the noise covariance matrix after the first matched filtering, i.e.,

$$
\boldsymbol{\Sigma}_{0}=\frac{\sigma^{2}}{\xi} \boldsymbol{I}+\omega \mathbb{E}\left[\boldsymbol{X}_{\beta} \boldsymbol{X}_{\beta}^{H}\right] .
$$

Define a set of random vectors $\hat{\boldsymbol{X}}_{t, n}=\boldsymbol{X}_{n}+\boldsymbol{\Sigma}_{t}^{\frac{1}{2}} \boldsymbol{V}_{n}$, $\forall n$, where $\boldsymbol{X}_{n} \in \mathbb{C}^{M \times 1}$ follows the distribution given in (11), and $\boldsymbol{V}_{n} \in \mathbb{C}^{M \times 1} \sim \mathcal{C N}(\mathbf{0}, \boldsymbol{I})$ is independent of $\boldsymbol{X}_{n}$. The state evolution analysis says that in the vector AMP algorithm, applying the denoiser to $\left(\boldsymbol{a}_{n}^{H} \boldsymbol{R}^{t}\right)^{H}+\boldsymbol{x}_{n}^{t}$ as shown in (13) is statistically equivalent to applying the denoiser to

$$
\hat{\boldsymbol{x}}_{t, n}=\boldsymbol{x}_{n}+\boldsymbol{\Sigma}_{t}^{\frac{1}{2}} \boldsymbol{v}_{n}=\alpha_{n} \boldsymbol{h}_{n}+\boldsymbol{\Sigma}_{t}^{\frac{1}{2}} \boldsymbol{v}_{n}, \quad \forall n,
$$

where the distributions of $\hat{\boldsymbol{x}}_{t, n}$ and $\boldsymbol{v}_{n}$ are captured by the random vectors $\hat{\boldsymbol{X}}_{t, n}$ and $\boldsymbol{V}_{n}$ [19], [26], [28].

\section{MMSE Denoiser Design for Vector AMP}

The key advantage of the equivalent signal model given in 17 is the decoupling of the estimation between different users, which allows us to design the denoiser $\eta_{t, n}(\cdot)$ in the vector AMP algorithm to minimize the MSE (12) based on the above decoupled signal model. Specifically, in the $t$ th iteration of the AMP algorithm, the MMSE denoiser $\eta_{t, n}(\cdot)$ is given by the conditional expectation $\mathbb{E}\left[\boldsymbol{X}_{n} \mid \hat{\boldsymbol{X}}_{t, n}\right]$. This denoiser has been derived in [18], [26], but is re-derived in Appendix $\mathrm{A}$ and expressed below in a form that highlights its structural dependence in $M$ :

$$
\begin{aligned}
\eta_{t, n}\left(\hat{\boldsymbol{x}}_{t, n}\right) & =\mathbb{E}\left[\boldsymbol{X}_{n} \mid \hat{\boldsymbol{X}}_{t, n}=\hat{\boldsymbol{x}}_{t, n}\right] \\
& =\phi_{t, n} \beta_{n}\left(\beta_{n} \boldsymbol{I}+\boldsymbol{\Sigma}_{t}\right)^{-1} \hat{\boldsymbol{x}}_{t, n}, \quad \forall t, n,
\end{aligned}
$$

where

$$
\begin{aligned}
\phi_{t, n} & =\frac{1}{1+\frac{1-\epsilon}{\epsilon} \exp \left(-M\left(\pi_{t, n}-\psi_{t, n}\right)\right)}, \\
\pi_{t, n} & =\frac{\hat{\boldsymbol{x}}_{t, n}^{H}\left(\boldsymbol{\Sigma}_{t}^{-1}-\left(\boldsymbol{\Sigma}_{t}+\beta_{n} \boldsymbol{I}\right)^{-1}\right) \hat{\boldsymbol{x}}_{t, n}}{M}, \\
\psi_{t, n} & =\frac{\log \operatorname{det}\left(\boldsymbol{I}+\beta_{n} \boldsymbol{\Sigma}_{t}^{-1}\right)}{M} .
\end{aligned}
$$

Examining the functional form of the MMSE denoiser (18)-(21), it is worthwhile to note that if all the users are active, i.e., $\epsilon=1$, it follows that $\phi_{t, n}=1, \forall n$, in which case the MMSE denoiser given in (18) reduces to the linear MMSE estimator: $\eta_{t, n}\left(\hat{\boldsymbol{x}}_{t, n}\right)=\beta_{n}\left(\beta_{n} \boldsymbol{I}+\boldsymbol{\Sigma}_{t}\right)^{-1} \hat{\boldsymbol{x}}_{t, n}$, which is widely used for channel training when the user activity 
is known [20]-[22]. When the device activity needs to be detected, the above MMSE denoiser is a non-linear function of $\hat{\boldsymbol{x}}_{t, n}$ due to the functional form of $\phi_{t, n}$.

\section{State Evolution for MMSE Denoiser Based Vector AMP}

The general state evolution of the AMP algorithm as in (15) applies to any arbitrary denoiser $\eta_{t, n}(\cdot)$. With the MMSE denoiser (18), the state evolution can be considerably simplified.

Theorem 1: Consider the MMSE denoiser based vector AMP algorithm for device detection and channel estimation in the asymptotic regime in which the number of users $N$, the number of active users $K$, and the length of the pilot sequences $L$ all go to infinity, while their ratios converge to some fixed positive values, i.e., $N / L \rightarrow \omega$ and $K / N \rightarrow \epsilon$ with $\omega, \epsilon \in(0, \infty)$, and while keeping the total transmit power $\xi$ fixed. The matrix $\boldsymbol{\Sigma}_{t}$ in the state evolution (15) always stays as a diagonal matrix with identical diagonal entries after each iteration, i.e.,

$$
\boldsymbol{\Sigma}_{t}=\tau_{t}^{2} \boldsymbol{I}, \quad \forall t \geq 0
$$

In this case, the signal model given in (17) reduces to

$$
\hat{\boldsymbol{x}}_{t, n}=\boldsymbol{x}_{n}+\tau_{t} \boldsymbol{v}_{n} \text {. }
$$

Moreover, the MMSE denoiser given in (18) reduces to

$$
\eta_{t, n}\left(\hat{\boldsymbol{x}}_{t, n}\right)=\phi_{t, n}\left(\frac{\beta_{n}}{\beta_{n}+\tau_{t}^{2}}\right) \hat{\boldsymbol{x}}_{t, n}, \quad \forall t, n,
$$

where $\phi_{t, n}$ is given in (19), while $\pi_{t, n}$ and $\psi_{t, n}$ are respectively given by

$$
\begin{aligned}
\pi_{t, n} & =\left(\frac{1}{\tau_{t}^{2}}-\frac{1}{\tau_{t}^{2}+\beta_{n}}\right) \frac{\hat{\boldsymbol{x}}_{t, n}^{H} \hat{\boldsymbol{x}}_{t, n}}{M}, \\
\psi_{t, n} & =\log \left(1+\frac{\beta_{n}}{\tau_{t}^{2}}\right) .
\end{aligned}
$$

At last, $\tau_{t}^{2}$ can be iteratively obtained as follows

$$
\begin{aligned}
& \tau_{0}^{2}=\frac{\sigma^{2}}{\xi}+\omega \epsilon \mathbb{E}_{\beta}[\beta], \\
& \tau_{t+1}^{2}=\frac{\sigma^{2}}{\xi}+\omega \epsilon \mathbb{E}_{\beta}\left[\frac{\beta \tau_{t}^{2}}{\beta+\tau_{t}^{2}}\right]+\omega \mathbb{E}_{\beta}\left[\vartheta_{t, \beta}\left(\tau_{t}^{2}\right)\right], t \geq 0
\end{aligned}
$$

with the term $\vartheta_{t, \beta}\left(\tau_{t}^{2}\right)$ expressed as

$\vartheta_{t, \beta}\left(\tau_{t}^{2}\right)=\frac{1}{M} \mathbb{E}_{\hat{\boldsymbol{X}}_{t, \beta}}\left[\phi_{t, \beta}\left(1-\phi_{t, \beta}\right) \frac{\beta^{2}}{\left(\beta+\tau_{t}^{2}\right)^{2}} \hat{\boldsymbol{X}}_{t, \beta}^{H} \hat{\boldsymbol{X}}_{t, \beta}\right]$.

Here, $\hat{\boldsymbol{X}}_{t, \beta}$ is the random vector that captures the distribution of the signal $\hat{\boldsymbol{x}}_{t, n}$ given in (23), and $\phi_{t, \beta}$ captures the distribution of $\phi_{t, n}$, which is implicitly a function of $\hat{\boldsymbol{x}}_{t, n}$.

Proof: Please refer to Appendix B

The key observation of Theorem 1 is that because each user's channels across the multiple receive antennas at the BS are assumed to be uncorrelated, the residual noise in (17) remains uncorrelated across the antennas. This is in spite of the fact that the vector AMP algorithm involves non-linear processing in $\phi_{t, n}$ as in (18). This scalar form of the state evolution significantly simplifies performance analysis of the device activity detection and channel estimation.

\section{E. Device Detection and Channel Estimation by Vector $A M P$}

We are now ready to state the proposed device activity detector and channel estimator. Observe that the functional form of $\phi_{t, n}$ as given in (18) is such that for large $M$, we have that $\phi_{t, n}$ tends to 1 if $\pi_{t, n}>\psi_{t, n}$ and 0 if $\pi_{t, n}<\psi_{t, n}$. As a result, the AMP algorithm suggests that it is reasonable to adopt a threshold strategy for user activity detection, i.e., to declare a user as active or not simply based on whether $\pi_{t, n}$ exceeds the threshold of $\psi_{t, n}$. Using (25)-(26) and the scalar form of the AMP state evolution, the proposed device activity detector and channel estimator are as follows.

Definition 1: The MMSE vector AMP algorithm based device activity detector is defined as the following thresholdbased detector. After $t$ iterations of AMP, compute $\tau_{t}^{2}$ according to (27)-28). For each user $n$, compare $\pi_{t, n}$ and $\psi_{t, n}$ as defined in 25)-26, i.e.,

$$
\begin{aligned}
& \varpi\left(\boldsymbol{x}_{n}^{t}, \boldsymbol{R}^{t}\right)= \\
& \begin{cases}1, & \text { if }\left(\left(\boldsymbol{R}^{t}\right)^{H} \boldsymbol{a}_{n}+\boldsymbol{x}_{n}^{t}\right)^{H}\left(\left(\boldsymbol{R}^{t}\right)^{H} \boldsymbol{a}_{n}+\boldsymbol{x}_{n}^{t}\right)>\theta_{t, n}, \\
0, & \text { if }\left(\left(\boldsymbol{R}^{t}\right)^{H} \boldsymbol{a}_{n}+\boldsymbol{x}_{n}^{t}\right)^{H}\left(\left(\boldsymbol{R}^{t}\right)^{H} \boldsymbol{a}_{n}+\boldsymbol{x}_{n}^{t}\right)<\theta_{t, n},\end{cases}
\end{aligned}
$$

with a threshold $\theta_{t, n}=M \log \left(1+\frac{\beta_{n}}{\tau_{t}^{2}}\right) /\left(\frac{1}{\tau_{t}^{2}}-\frac{1}{\tau_{t}^{2}+\beta_{n}}\right)$. Further, given that a device $k$ is declared active, its channel is estimated as:

$$
\hat{\boldsymbol{h}}_{t, k}=\boldsymbol{x}_{k}^{t} .
$$

We remark that the MMSE denoiser given in 24) is a scaled version of the observation. As a result, the complexity of the vector AMP algorithm introduced in (13) and (14) mainly comes from the matrix multiplication $\boldsymbol{A X}^{t+1}$. Since $\boldsymbol{A} \in \mathbb{C}^{L \times N}$ and $\boldsymbol{X}^{t+1} \in \mathbb{C}^{N \times M}$, the complexity of the AMP algorithm is in the order of $\mathcal{O}(L N M)$ per iteration.

\section{Performance of Vector Amp Based Detector}

In this section, we analyze the performance of the above MMSE vector AMP based device activity detector in terms of missed detection and false alarm error probabilities, as well as the associated channel estimation error based on the state evolution, as functions of the number of receive antennas $M$ at the BS. The results of this section pertain to finite $M$. Asymptotic result with $M$ going to infinity is presented in the next section.

\section{A. Probabilities of Missed Detection and False Alarm}

We are ready to examine the error probability for the MMSE vector AMP based detector as defined in (30). Let the missed detection and false alarm probabilities of user $n$ 
after the $t$ th iteration of the MMSE denoiser based AMP algorithm be defined as

$$
P_{t, n}^{\mathrm{MD}}(M)=\operatorname{Pr}\left(\varpi\left(\boldsymbol{x}_{n}^{t}, \boldsymbol{R}^{t}\right)=0 \mid \alpha_{n}=1\right),
$$

and

$$
P_{t, n}^{\mathrm{FA}}(M)=\operatorname{Pr}\left(\varpi\left(\boldsymbol{x}_{n}^{t}, \boldsymbol{R}^{t}\right)=1 \mid \alpha_{n}=0\right),
$$

respectively, as functions of $M$, the number of antennas at the BS. The following theorem characterizes $P_{t, n}^{\mathrm{MD}}(M)$ and $P_{t, n}^{\mathrm{FA}}(M)$ analytically in terms of $\tau_{t}^{2}$ and $M$.

Theorem 2: Consider the device activity detector for massive connectivity (30), based on the vector AMP algorithm with MMSE denoiser. Fix the number of BS receive antennas $M$, and consider the asymptotic regime in which the number of users $N$, the number of active users $K$, and the length of the pilot sequences $L$ all go to infinity, while their ratios converge to some fixed positive values, i.e., $N / L \rightarrow \omega$ and $K / N \rightarrow \epsilon$ with $\omega, \epsilon \in(0, \infty)$, and while keeping the total transmit power $\xi$ fixed. After $t$ iterations of the AMP algorithm, the probabilities of missed detection and false alarm of user $n$ can be expressed, respectively, as

$$
P_{t, n}^{\mathrm{MD}}(M)=\frac{\Gamma\left(M, b_{t, n} M\right)}{\Gamma(M)},
$$

and

$$
P_{t, n}^{\mathrm{FA}}(M)=\frac{\bar{\Gamma}\left(M, c_{t, n} M\right)}{\Gamma(M)},
$$

where $\Gamma(\cdot), \underline{\Gamma}(\cdot, \cdot)$, and $\bar{\Gamma}(\cdot, \cdot)$ denote the Gamma function, lower incomplete Gamma function, and upper incomplete Gamma function [29], respectively, and

$$
\begin{aligned}
b_{t, n} & =\frac{\tau_{t}^{2}}{\beta_{n}} \log \left(1+\frac{\beta_{n}}{\tau_{t}^{2}}\right), \\
c_{t, n} & =\left(\frac{\beta_{n}+\tau_{t}^{2}}{\beta_{n}}\right) \log \left(1+\frac{\beta_{n}}{\tau_{t}^{2}}\right),
\end{aligned}
$$

and $\tau_{t}^{2}$ is given by 27 28 .

Proof: Please refer to Appendix C

The above analysis of detection probabilities of error hinges upon the Gaussian signal model of the AMP state evolution (17). As consequence of the scalar signal model (23), the proposed device activity detector 30 becomes a threshold detector on a $\chi^{2}$-distribution as defined in 25). This allows both missed detection and false alarm probabilities to be characterized analytically.

An important observation is that due to the fact that $a>\log (1+a)>\frac{a}{1+a}$ for $a>0$, we have that $b_{t, n}<1$ and $c_{t, n}>1$. As result, one can show that both error probabilities eventually go to zero as $M \rightarrow \infty$. This asymptotic behavior is explored in more detail in the next section.

Finally, we note that at the convergence of the AMP algorithm, $\tau_{t}^{2}$ converges to the fixed-point solution to (28), i.e., $\tau_{\infty}^{2}$. The detection error probabilities may then be expressed as 34- 35 with $\tau_{t}^{2}$ replaced by $\tau_{\infty}^{2}$.

\section{B. Analysis of Channel Estimation Error}

The state evolution analysis of AMP further allows us to evaluate the channel estimation performance. For any active user $k \in \mathcal{K}$, the estimated channel $\hat{\boldsymbol{h}}_{t, k}$ is as defined in (31), with $\Delta \boldsymbol{h}_{t, k}=\boldsymbol{h}_{t, k}-\hat{\boldsymbol{h}}_{t, k}$ denoting the corresponding channel estimation error. The following theorem characterizes the covariance matrices of the estimated channels and the channel estimation errors of the active users as function of the number of BS antennas $M$.

Theorem 3: Consider the channel estimator 31 for massive connectivity, based on the vector AMP algorithm with MMSE denoiser. Fix the number of BS receive antennas $M$, and consider the asymptotic regime in which the number of users $N$, the number of active users $K$, and the length of the pilot sequences $L$ all go to infinity, while their ratios converge to some fixed positive values, i.e., $N / L \rightarrow \omega$ and $K / N \rightarrow \epsilon$ with $\omega, \epsilon \in(0, \infty)$, and while keeping the total transmit power $\xi$ fixed. For each active user $k \in \mathcal{K}$, the covariance matrices of its estimated channel and channel estimation error are given, respectively, by

$$
\begin{aligned}
& \operatorname{Cov}\left(\hat{\boldsymbol{h}}_{t, k}, \hat{\boldsymbol{h}}_{t, k}\right)=v_{t, k}(M) \boldsymbol{I}, \\
& \operatorname{Cov}\left(\Delta \boldsymbol{h}_{t, k}, \Delta \boldsymbol{h}_{t, k}\right)=\Delta v_{t, k}(M) \boldsymbol{I},
\end{aligned}
$$

where $v_{t, k}(M)$ and $\Delta v_{t, k}(M)$ are given in (40) and 41) at the bottom of the page, where the expectation is over both the channel $\boldsymbol{h}_{k}$ and the residual noise $\boldsymbol{v}_{k}$ modeled as Gaussian random variables, and $\tau_{t}^{2}$ is given by state evolution (27)-28.

Proof: Please refer to Appendix D

As already noted earlier, at the convergence of the AMP algorithm, $\tau_{t}^{2}$ converges to $\tau_{\infty}^{2}$. Then, the channel estimation error converges to 38 (39) with $\tau_{t}^{2}$ replaced by $\tau_{\infty}^{2}$.

\section{Asymptotic Performance With Massive Mimo}

We have so far characterized the probabilities of missed detection and false alarm as well as the covariance matrices of the estimated channels and channel estimation errors of the proposed MMSE denoiser based AMP detector in the asymptotic regime where $N, K, L$ all go to infinity with fixed ratios, but with fixed $M$. In this section, we further let

$$
\begin{aligned}
& v_{t, k}(M)=\frac{1}{M} \mathbb{E}\left[\phi_{t, k}^{2}\left(\frac{\beta_{k}}{\beta_{k}+\tau_{t}^{2}}\right)^{2}\left(\boldsymbol{h}_{k}+\tau_{t} \boldsymbol{v}_{k}\right)^{H}\left(\boldsymbol{h}_{k}+\tau_{t} \boldsymbol{v}_{k}\right)\right] \\
& \Delta v_{t, k}(M)=\frac{1}{M} \mathbb{E}\left[\left(\phi_{t, k} \frac{\beta_{k}}{\beta_{k}+\tau_{t}^{2}}\left(\boldsymbol{h}_{k}+\tau_{t} \boldsymbol{v}_{k}\right)-\boldsymbol{h}_{k}\right)^{H}\left(\phi_{t, k} \frac{\beta_{k}}{\beta_{k}+\tau_{t}^{2}}\left(\boldsymbol{h}_{k}+\tau_{t} \boldsymbol{v}_{k}\right)-\boldsymbol{h}_{k}\right)\right] .
\end{aligned}
$$


$M$ go to infinity and study the asymptotic massive MIMO regime to gain further insight.

\section{A. Asymptotic Performance of User Activity Detection}

A main result of this paper is to show that the proposed device activity detector performs well in the massive MIMO regime. The intuitive reason behind this result is that the threshold detection (30) involves a comparison between $\pi_{t, n}$ as in 25) with $\psi_{t, n}$ as in 26. According to the AMP state evolution signal model (23), as $M \rightarrow \infty$, by the law of large numbers, we have

$$
\pi_{t, n} \rightarrow \begin{cases}\left(\frac{1}{\tau_{t}^{2}}-\frac{1}{\tau_{t}^{2}+\beta_{n}}\right)\left(\tau_{t}^{2}+\beta_{n}\right) & \text { if } \alpha_{n}=1 \\ \left.\frac{1}{\tau_{t}^{2}}-\frac{1}{\tau_{t}^{2}+\beta_{n}}\right) \tau_{t}^{2} & \text { if } \alpha_{n}=0\end{cases}
$$

which simplifies to $\pi_{t, n} \rightarrow \beta_{n} / \tau_{t}^{2}$ if user $n$ is active, and $\pi_{t, n} \rightarrow \beta_{n} /\left(\tau_{t}^{2}+\beta_{n}\right)$ if it is not. Now compare with $\psi_{t, n}=$ $\log \left(1+\beta_{n} / \tau_{t}^{2}\right)$. Using the fact that $a>\log (1+a)>\frac{a}{1+a}$ for all $a>0$, we always have

$$
\frac{\beta_{n}}{\tau_{t}^{2}}>\log \left(1+\frac{\beta_{n}}{\tau_{t}^{2}}\right)>\frac{\beta_{n}}{\tau_{t}^{2}+\beta_{n}}
$$

as long as $\beta_{n}>0$ and $\tau_{t}^{2}<\infty$. Thus, we have that asymptotically as $M \rightarrow \infty$, it is always true that $\pi_{t, n}>\psi_{t, n}$ when $\alpha_{n}=1$ and $\pi_{t, n}<\psi_{t, n}$ when $\alpha_{n}=0$. In other words, the proposed detector always makes the correct decision in the massive MIMO regime.

The above argument can be made more precise by utilizing the analytic probabilities of missed detection and false alarm expressions as characterized in Theorem 2 to show that the error probabilities actually go down exponentially in $M$.

Theorem 4: The probabilities of missed detection and false alarm for any user $n$ after $t$ iterations of AMP algorithm, as characterized in (34) and (35) for the MMSE denoiser based device activity detector, scale in $M$, the number of receive antennas at the BS, as follows:

$$
\begin{aligned}
P_{t, n}^{\mathrm{MD}}(M)=-\frac{\exp \left(-\frac{1}{2} M \nu_{t, n}^{2}\right)}{2 \sqrt{2 \pi M}}\left(\frac{1}{b_{t, n}-1}+\frac{1}{\nu_{t, n}}\right) \\
+o\left(\frac{\exp (-M)}{\sqrt{M}}\right),
\end{aligned}
$$

and

$$
\begin{aligned}
P_{t, n}^{\mathrm{FA}}(M)=\frac{\exp \left(-\frac{1}{2} M \varsigma_{t, n}^{2}\right)}{2 \sqrt{2 \pi M}}\left(\frac{1}{c_{t, n}-1}+\frac{1}{\varsigma_{t, n}}\right) \\
+o\left(\frac{\exp (-M)}{\sqrt{M}}\right),
\end{aligned}
$$

where $b_{t, n}$ and $c_{t, n}$ are as defined in (36) and (37) respectively, and

$$
\begin{aligned}
& \nu_{t, n}=-\sqrt{2\left(b_{t, n}-1-\log b_{t, n}\right)}, \\
& \varsigma_{t, n}=\sqrt{2\left(c_{t, n}-1-\log c_{t, n}\right)} .
\end{aligned}
$$

Further, assuming that $\beta_{n}$ is bounded below, i.e., $\beta_{n}>$ $\beta_{\text {min }}, \forall n$, we then have $b_{t, n} \leq 1-\varepsilon_{t, n}^{(1)}, c_{t, n} \geq 1+\varepsilon_{t, n}^{(2)}$, $\nu_{t, n} \leq-\varepsilon_{t, n}^{(3)}, \varsigma_{t, n} \geq \varepsilon_{t, n}^{(4)}$, for some positive constants $\varepsilon_{t, n}^{(1)}$, $\varepsilon_{t, n}^{(2)}, \varepsilon_{t, n}^{(3)}$, and $\varepsilon_{t, n}^{(4)}$ that are independent of $M$, and that

$$
\lim _{M \rightarrow \infty} P_{t, n}^{\mathrm{MD}}(M)=\lim _{M \rightarrow \infty} P_{t, n}^{\mathrm{FA}}(M)=0, \quad \forall t, n .
$$

Proof: Please refer to Appendix E

It is interesting to note that Theorem 4 shows that the device detection error probability goes to zero as $M \rightarrow \infty$ for any arbitrary $t$. Thus remarkably, this is true even for $t=1$, i.e., with infinitely large $M$, detection is already correct after just one iteration. Further, it is also true for when $t \rightarrow \infty$.

Theorem 4 is also striking in stating that accurate user activity detection is guaranteed as long as $M$ is large, regardless of the relative ratios between $N, K, L$. Thus, this is true even if $L \leq K$. However as shown later in the paper, channel estimation performance would be poor in this $L \leq K$ case.

\section{B. State Evolution in the Asymptotic Massive MIMO Regime}

Theorem 4 states that when $M \rightarrow \infty$, the detection strategy (30) is almost always successful. This also implies that the MMSE denoiser in the AMP algorithm (19) must converge as follows

$$
\phi_{t, n} \rightarrow \begin{cases}1, & \text { if } \alpha_{n}=1, \\ 0, & \text { if } \alpha_{n}=0 .\end{cases}
$$

Using this fact, the state evolution equation of the AMP algorithm can be significantly simplified. Intuitively, since $\phi_{t, n}$ almost surely converges to 0 or 1 in the massive MIMO regime, the term $\vartheta_{t, \beta_{n}}(M)$ in [29], which captures the cost of imperfect user activity detection, becomes negligible when $M$ goes to infinity.

Theorem 5: For any user $n$ and at $t$ th iteration, as the number of antennas at the BS $M$ goes to infinity, we have

$$
\lim _{M \rightarrow \infty} \vartheta_{t, \beta}(M) \rightarrow 0
$$

Proof: Please refer to Appendix $\mathrm{F}$

As a consequence, as $M$ goes to infinity, the scalar form of the state evolution for the MMSE denoiser based AMP algorithm given in (28) reduces to

$$
\tau_{t+1}^{2}=\frac{\sigma^{2}}{\xi}+\omega \epsilon \mathbb{E}_{\beta}\left[\frac{\beta \tau_{t}^{2}}{\beta+\tau_{t}^{2}}\right],
$$

in the massive MIMO regime. It is reasonable to deduce that the fixed-point solution to the state evolution given in 28 approaches the fixed-point solution to the simplified state evolution shown in (50).

\section{Channel Estimation in Asymptotic Massive MIMO Regime}

As another consequence of (48), the covariance matrices of the estimated channels and the corresponding channel estimation errors as given in (38)-(39) can be considerably simplified in the massive MIMO regime when $M \rightarrow \infty$. 
Theorem 6: Assuming simplified state evolution (50) in the massive MIMO regime as $M$, the number of antennas at the BS, goes to infinity, for each active user $k \in \mathcal{K}$ and after the $t$ th iteration, the covariance matrices of the estimated channel and channel estimation error are as given in (38) and (39), where $v_{t, k}(M)$ and $\Delta v_{t, k}(M)$ respectively converge to

$$
\begin{aligned}
& \lim _{M \rightarrow \infty} v_{t, k}(M)=\frac{\beta_{k}^{2}}{\beta_{k}+\tau_{t}^{2}}, \\
& \lim _{M \rightarrow \infty} \Delta v_{t, k}(M)=\frac{\beta_{k} \tau_{t}^{2}}{\beta_{k}+\tau_{t}^{2}} .
\end{aligned}
$$

Proof: Please refer to Appendix $\mathrm{G}$

This result enables us to characterize the achievable rates of the massive connectivity system in the massive MIMO regime in Part II of this paper [5].

\section{Numerical ExAmples}

In this section, we provide numerical examples to verify the results of this paper. The setup is as follows. There are $N=2,000$ devices in the cell, and at any time slot, each user accesses the channel with a probability of $\epsilon=0.05$. Let $d_{n}$ denote the distance between user $n$ and the BS, $\forall n$. It is assumed that $d_{n}$ 's are randomly distributed in the regime $[0.05 \mathrm{~km}, 1 \mathrm{~km}]$. The path loss model of the wireless channel for user $n$ is given as $\beta_{n}=-128.1-36.7 \log _{10}\left(d_{n}\right)$ in $\mathrm{dB}$, $\forall n$. The bandwidth and the coherence time of the wireless channel are $1 \mathrm{MHz}$ and $1 \mathrm{~ms}$, respectively, and thus in each coherence block $T=1000$ symbols can be transmitted. The transmit power for each user at both the first and second transmission phases is $\rho^{\text {pilot }}=23 \mathrm{dBm}$. The power spectral density of the AWGN at the BS is assumed to be $-169 \mathrm{dBm} / \mathrm{Hz}$. Moreover, the numerical results here are obtained by averaging over $10^{7}$ channel realizations.

\section{A. Probabilities of Missed Detection and False Alarm}

First, we examine the performance of user activity detection achieved by the proposed AMP-based detector. Given any $M$, define the average probabilities of missed detection and false alarm over all users as $P^{\mathrm{MD}}(M)=$ $\sum_{n=1}^{N} P_{\infty, n}^{\mathrm{MD}}(M) / N$ and $P^{\mathrm{FA}}(M)=\sum_{n=1}^{N} P_{\infty, n}^{\mathrm{FA}}(M) / N$, respectively, where $P_{\infty, n}^{\mathrm{MD}}(M)$ and $P_{\infty, n}^{\mathrm{FA}}(M)$ denote the probabilities of missed detection and false alarm of user $n$ after the convergence of the AMP algorithm as defined in (32) and (33). Fig. 3 shows $P^{\mathrm{MD}}(M)$ and $P^{\mathrm{FA}}(M)$ versus $M$, the number of antennas at the BS, when the length of the user pilot sequences is $L=90$ both in simulation and as predicted by Theorem 4 First, it is observed that the probabilities of missed detection and false alarm characterized in Theorem 4 match the numerical results from AMP algorithm very well. Next, it is observed that as $M$ increases, both $P^{\mathrm{MD}}(M)$ and $P^{\mathrm{FA}}(M)$ decrease exponentially fast towards zero as predicated by Theorem 4

Fig. 4 shows the probabilities of missed detection and false alarm as functions of $M$ for various values of $L$. It is observed that although for any $L$, both $P^{\mathrm{MD}}(M)$ and

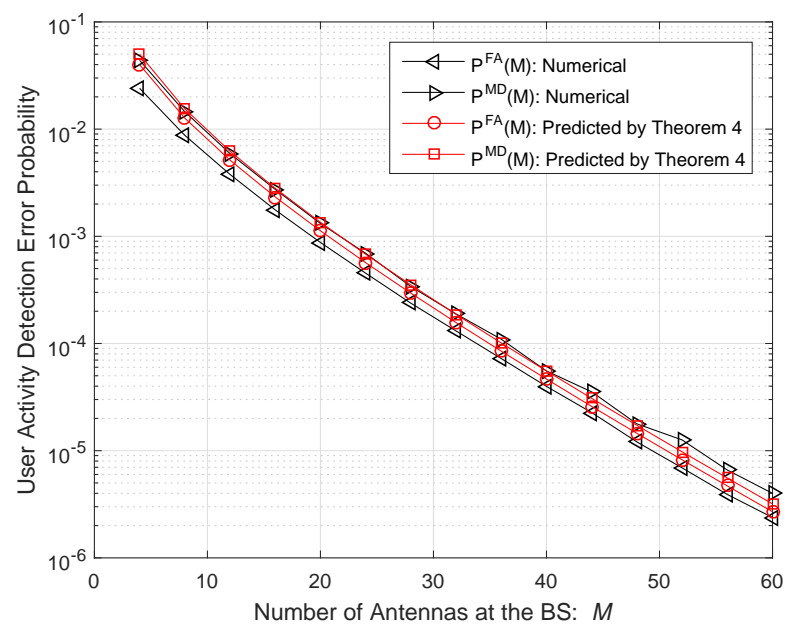

Fig. 3. Numerical simulations versus analytical expressions of probabilities of missed detection and false alarm as functions of the number of antennas at the BS when each of the $N=2000$ users accesses the channel with probability $\epsilon=0.05$ at each coherence time. The transmit power of each user is $\rho^{\text {pilot }}=23 \mathrm{dBm}$. The pilot sequence length is $L=90$.

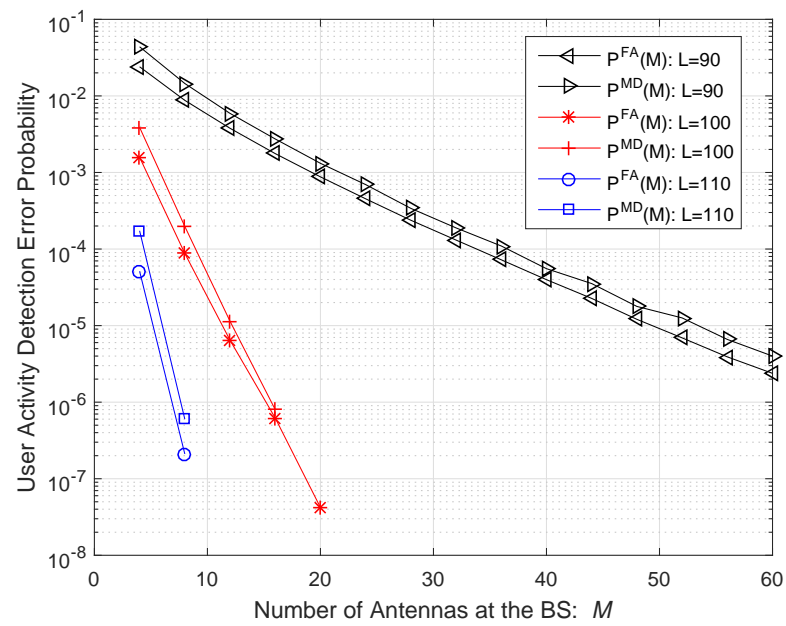

Fig. 4. Probabilities of missed detection and false alarm as functions of the number BS antennas when each of the $N=2000$ users accesses the channel with probability $\epsilon=0.05$ at each coherence time. The transmit power of the users is $\rho^{\text {pilot }}=23 \mathrm{dBm}$.

$P^{\mathrm{FA}}(M)$ decrease over $M$, but the reduction is more rapid when $L$ is 110 as compared to 90 . Specifically, when $L=$ $90<K, M=52$ antennas are needed to drive $P^{\mathrm{MD}}(M)$ and $P^{\mathrm{FA}}(M)$ below $10^{-5}$; when $L=110>K$, only $M=$ 8 antennas are sufficient. The point is that although Theorem 4 holds for all $L$ as long as $M \rightarrow \infty$, in practice the pilot length $L$ should be chosen to be larger than $K \approx N \epsilon=$ 100 , as otherwise very large number of antennas would be needed.

Fig. 5 shows the probabilities of missed detection and false alarm versus the transmit power of the users $\rho^{\text {pilot }}$, with different numbers of antennas at the BS, where the 


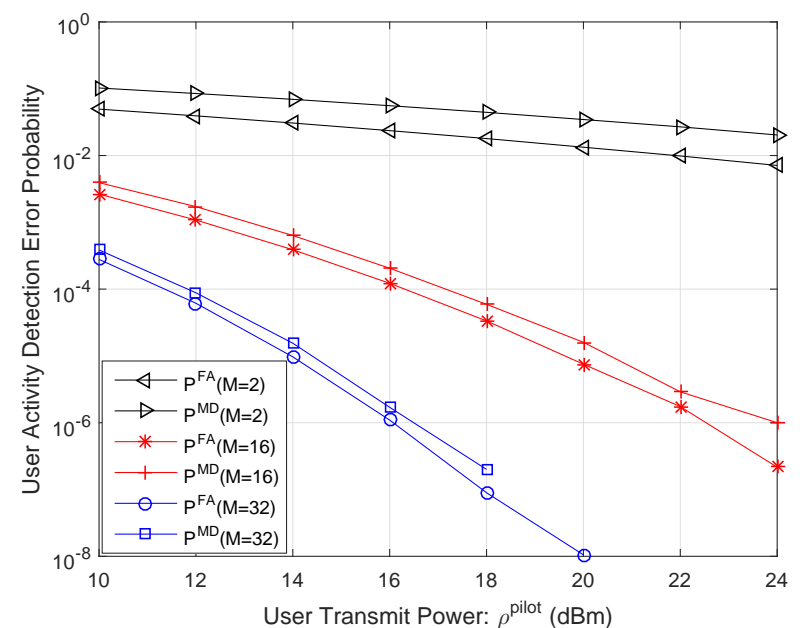

Fig. 5. Probabilities of missed detection and false alarm as functions of the identical transmit power of all the active users with different numbers of antennas at the BS when each of the $N=2000$ users accesses the channel with probability $\epsilon=0.05$ at each coherence time and the pilot sequence length is $L=N \epsilon=100$.

length of the user pilot sequences is set to be $L=100$. It is observed that for all values of $\rho^{\text {pilot }}$, when there are $M=32$ antennas at the $\mathrm{BS}$, both $P^{\mathrm{MD}}$ and $P^{\mathrm{FA}}$ are significantly lower than those in the cases when there are $M=2$ and $M=16$ antennas at the BS. Further, when $M=32, P^{\mathrm{MD}}$ and $P^{\mathrm{FA}}$ decrease much faster with $\rho^{\text {pilot }}$ than the cases of $M=2$ and $M=16$.

Fig. 6 shows the probabilities of missed detection and false alarm versus the length of the pilot sequences, $L$, with different number of antennas at the BS, where the transmit power of each user is $23 \mathrm{dBm}$, and the number of antennas at the $\mathrm{BS}$ is $M=4,8$ or 16 . It is observed that both $P^{\mathrm{MD}}$ and $P^{\mathrm{FA}}$ decrease as the pilot sequence length $L$ increases and when $M$ increases from 4 to 8 and 16 .

\section{B. Channel Estimation Error}

Fig. 7 verifies the channel estimation error as predicted by Theorem 6 after the convergence of the AMP algorithm. In particular, we select an active user $k$ that is $0.8 \mathrm{~km}$ away from the BS and run AMP to calculate the covariance matrices of its estimated channel and corresponding channel estimation error numerically, for two cases of when the BS has $M=16$ or $M=64$ antennas. It is observed that when $M=64$, both $\nu_{\infty, k}$ and $\Delta \nu_{\infty, k}$ obtained numerically from the AMP algorithm perfectly match those predicated by Theorem 6 for all values of $L$. When $M=16$, there is some mismatch in the regime of $L<90$ as device detection is not perfect in the regime where $M$ and $L$ are both small. We remark that to have reasonable channel estimation error, the AMP should operate in the regime of $L>K \approx N \epsilon$.

\section{CONCLUSION}

Device activity detection and channel estimation are crucial components for wireless massive connectivity applica-

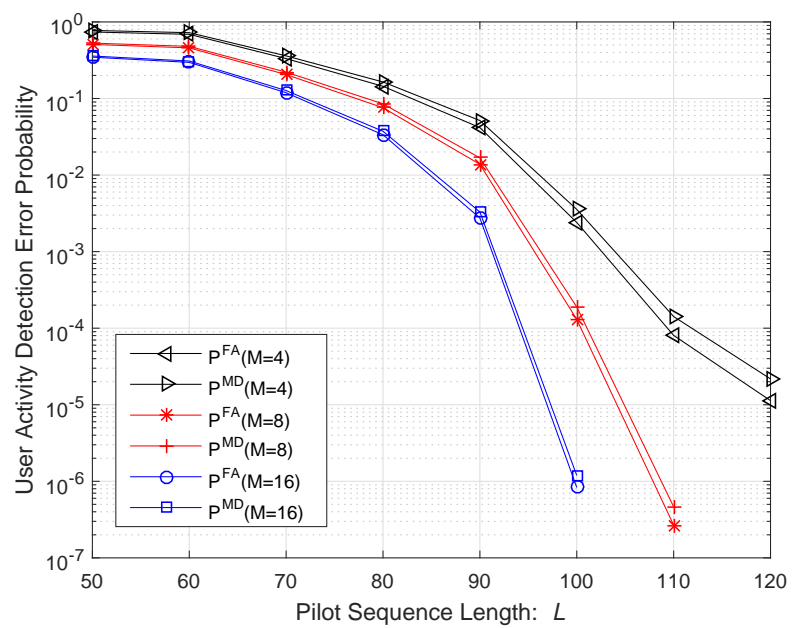

Fig. 6. Probabilities of missed detection and false alarm as functions of the pilot sequence lengths when each of the $N=2000$ users accesses the channel with probability $\epsilon=0.05$ at each coherence time and the transmit power of users is $\rho^{\text {pilot }}=23 \mathrm{dBm}$. Further, the BS is equipped with $M=4,8$ or 16 antennas.

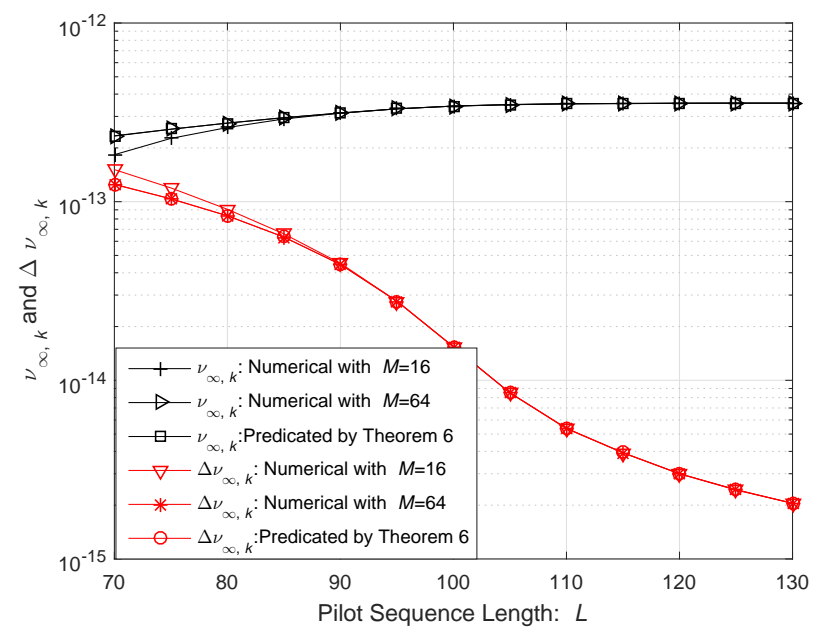

Fig. 7. Variance of the estimated channel and the channel estimation error for a particular user at $0.8 \mathrm{~km}$ from the BS as function of pilot sequence length. Here, each of the $N=2000$ users accesses the channel with probability $\epsilon=0.05$ in each coherence time; the BS has $M=16$ or 64 antennas.

tions. This paper shows that the vector AMP algorithm is a natural tool for sparse activity detection. Utilizing the state evolution analysis, both the missed detection and false alarm probabilities and channel estimation error can be analytically characterized. Further, in the massive MIMO regime, perfect device activity detection can be guaranteed, but channel estimation error remains. Part II of this paper [5] utilizes these results to further characterize the achievale rate. 


\section{APPENDIX}

\section{A. Derivation of MMSE Denoiser (18)}

Lemma 1: Let $\hat{\boldsymbol{X}}=\boldsymbol{X}+\boldsymbol{\Sigma}^{\frac{1}{2}} \boldsymbol{V}$ where $\boldsymbol{X} \in \mathbb{C}^{M \times 1}$ has a Bernoulli-Gaussian distribution $(1-\epsilon) \delta_{0}+\epsilon p_{\boldsymbol{h}_{\beta}}$, with $\boldsymbol{h}_{\beta} \sim \mathcal{C N}(\mathbf{0}, \beta \boldsymbol{I}), \boldsymbol{\Sigma}$ is some positive definite matrix and $\boldsymbol{V} \in \mathbb{C}^{M \times 1} \sim \mathcal{C N}(\mathbf{0}, \boldsymbol{I})$ is independent of $\boldsymbol{X}$. Define

$\phi(\hat{\boldsymbol{x}})=$

$\overline{1+\frac{1-\epsilon}{\epsilon} \exp \left(-\hat{\boldsymbol{x}}^{H}\left(\boldsymbol{\Sigma}^{-1}-(\boldsymbol{\Sigma}+\beta \boldsymbol{I})^{-1}\right) \hat{\boldsymbol{x}}\right)\left|\boldsymbol{I}+\beta \boldsymbol{\Sigma}^{-1}\right|}$

Then,

$$
\mathbb{E}[\boldsymbol{X} \mid \hat{\boldsymbol{X}}=\hat{\boldsymbol{x}}]=\phi(\hat{\boldsymbol{x}}) \beta(\beta \boldsymbol{I}+\boldsymbol{\Sigma})^{-1} \hat{\boldsymbol{x}},
$$

and

$$
\begin{aligned}
\mathbb{E}\left[\boldsymbol{X} \boldsymbol{X}^{H} \mid \hat{\boldsymbol{X}}=\hat{\boldsymbol{x}}\right]=\phi(\hat{\boldsymbol{x}})\left(\beta \boldsymbol{I}-\beta^{2}(\beta \boldsymbol{I}+\boldsymbol{\Sigma})^{-1}\right. \\
\left.+\beta^{2}(\beta \boldsymbol{I}+\boldsymbol{\Sigma})^{-1} \hat{\boldsymbol{x}} \hat{\boldsymbol{x}}^{H}(\beta \boldsymbol{I}+\boldsymbol{\Sigma})^{-1}\right)
\end{aligned}
$$

Proof: For convenience, define $\overline{\boldsymbol{X}}=\boldsymbol{h}_{\beta}+\boldsymbol{\Sigma}^{\frac{1}{2}} \boldsymbol{V}$. Let $\hat{\boldsymbol{X}}=\overline{\boldsymbol{X}}$ with probability $\epsilon$ and $\hat{\boldsymbol{X}}=\boldsymbol{\Sigma}^{\frac{1}{2}} \boldsymbol{V}$ with probability $1-\epsilon$. By standard estimation theory, we have

$$
\mathbb{E}\left[\boldsymbol{h}_{\beta} \mid \overline{\boldsymbol{X}}=\overline{\boldsymbol{x}}\right]=\beta(\beta \boldsymbol{I}+\boldsymbol{\Sigma})^{-1} \overline{\boldsymbol{x}},
$$

and

$$
\begin{aligned}
\mathbb{E}\left[\boldsymbol{h}_{\beta} \boldsymbol{h}_{\beta}^{H} \mid \overline{\boldsymbol{X}}=\right. & \overline{\boldsymbol{x}}]=\beta \boldsymbol{I}-\beta^{2}(\beta \boldsymbol{I}+\boldsymbol{\Sigma})^{-1} \\
& +\beta^{2}(\beta \boldsymbol{I}+\boldsymbol{\Sigma})^{-1} \overline{\boldsymbol{x}} \overline{\boldsymbol{x}}^{H}(\beta \boldsymbol{I}+\boldsymbol{\Sigma})^{-1}
\end{aligned}
$$

We now characterize $\mathbb{E}[\boldsymbol{X} \mid \hat{\boldsymbol{X}}=\hat{\boldsymbol{x}}]$ and $\mathbb{E}\left[\boldsymbol{X} \boldsymbol{X}^{H} \mid \hat{\boldsymbol{X}}=\hat{\boldsymbol{x}}\right]$ based on $\mathbb{E}\left[\boldsymbol{h}_{\beta} \mid \overline{\boldsymbol{X}}=\overline{\boldsymbol{x}}\right]$ and $\mathbb{E}\left[\boldsymbol{h}_{\beta} \boldsymbol{h}_{\beta}^{H} \mid \overline{\boldsymbol{X}}=\overline{\boldsymbol{x}}\right]$.

$$
\begin{aligned}
& \mathbb{E}[\boldsymbol{X} \mid \hat{\boldsymbol{X}}=\hat{\boldsymbol{x}}]=\int \boldsymbol{x} p_{\boldsymbol{X} \mid \hat{\boldsymbol{X}}}(\boldsymbol{X}=\boldsymbol{x} \mid \hat{\boldsymbol{X}}=\hat{\boldsymbol{x}}) d \boldsymbol{x} \\
& =\frac{1}{p_{\hat{\boldsymbol{X}}}(\hat{\boldsymbol{X}}=\hat{\boldsymbol{x}})} \int \boldsymbol{x} p_{\hat{\boldsymbol{X}} \mid \boldsymbol{X}}(\hat{\boldsymbol{X}}=\hat{\boldsymbol{x}} \mid \boldsymbol{X}=\boldsymbol{x}) \\
& \left(\epsilon{ }^{p} \boldsymbol{h}_{\beta}\left(\boldsymbol{h}_{\beta}=\boldsymbol{x}\right)+(1-\epsilon) \delta_{0}(\boldsymbol{x})\right) d \boldsymbol{x} \\
& =\frac{\epsilon}{p_{\hat{\boldsymbol{X}}}(\hat{\boldsymbol{X}}=\hat{\boldsymbol{x}})} \int \boldsymbol{x} p_{\overline{\boldsymbol{X}}, \boldsymbol{h}_{\beta}}\left(\overline{\boldsymbol{X}}=\hat{\boldsymbol{x}} \mid \boldsymbol{h}_{\beta}=\boldsymbol{x}\right) \\
& p_{\boldsymbol{h}_{\beta}}\left(\boldsymbol{h}_{\beta}=\boldsymbol{x}\right) d \boldsymbol{x} \\
& =\frac{\epsilon p \overline{\boldsymbol{X}}^{(\overline{\boldsymbol{X}}=\hat{\boldsymbol{x}})}}{p_{\hat{\boldsymbol{X}}}(\hat{\boldsymbol{X}}=\hat{\boldsymbol{x}})} \mathbb{E}\left[\boldsymbol{h}_{\beta} \mid \overline{\boldsymbol{X}}=\hat{\boldsymbol{x}}\right] \\
& =\phi(\hat{\boldsymbol{x}}) \beta(\beta \boldsymbol{I}+\boldsymbol{\Sigma})^{-1} \hat{\boldsymbol{x}},
\end{aligned}
$$

where (60) is because given $\boldsymbol{X}=\boldsymbol{x}, p_{\hat{\boldsymbol{X}} \mid \boldsymbol{X}}(\hat{\boldsymbol{X}}=\hat{\boldsymbol{x}} \mid \boldsymbol{X}=$ $\boldsymbol{x})$ is just the distribution of the random vector $\boldsymbol{x}+\boldsymbol{\Sigma}^{\frac{1}{2}} \boldsymbol{V}$, but the same is true for $p_{\overline{\boldsymbol{X}} \mid \boldsymbol{h}_{\beta}}\left(\overline{\boldsymbol{X}}=\hat{\boldsymbol{x}} \mid \boldsymbol{h}_{\beta}=\boldsymbol{x}\right)$, and (62) is because $p_{\hat{\boldsymbol{X}}}(\hat{\boldsymbol{X}}=\hat{\boldsymbol{x}})$ takes the form of

$$
\epsilon \frac{\exp \left(-\hat{\boldsymbol{x}}^{H}(\beta \boldsymbol{I}+\boldsymbol{\Sigma})^{-1} \hat{\boldsymbol{x}}\right)}{\pi^{M}|\beta \boldsymbol{I}+\boldsymbol{\Sigma}|}+(1-\epsilon) \frac{\exp \left(-\hat{\boldsymbol{x}}^{H} \boldsymbol{\Sigma}^{-1} \hat{\boldsymbol{x}}\right)}{\pi^{M}|\boldsymbol{\Sigma}|}
$$

so a straightforward computation gives that

$$
\frac{\epsilon p_{\hat{\boldsymbol{X}}}(\overline{\boldsymbol{X}}=\hat{\boldsymbol{x}})}{p_{\hat{\boldsymbol{X}}}(\hat{\boldsymbol{X}}=\hat{\boldsymbol{x}})}=\phi(\hat{\boldsymbol{x}}) .
$$

Similarly, it can be shown that $\mathbb{E}\left[\boldsymbol{X} \boldsymbol{X}^{H} \mid \hat{\boldsymbol{X}}=\hat{\boldsymbol{x}}\right]=$ $\phi(\hat{\boldsymbol{x}}) \mathbb{E}\left[\boldsymbol{h}_{\beta} \boldsymbol{h}_{\beta}^{H} \mid \overline{\boldsymbol{X}}=\hat{\boldsymbol{x}}\right]$.

\section{B. Proof of Theorem 1}

We first characterize the MSE term in (15) when the MMSE denoiser (18) is used in the vector AMP algorithm, then show that the state $\boldsymbol{\Sigma}_{t}$ stays as a diagonal matrix with identical diagonal entries throughout the AMP iterations.

Let $\hat{\boldsymbol{X}}_{t, \beta}=\boldsymbol{X}_{\beta}+\Sigma_{t}^{\frac{1}{2}} \boldsymbol{V}$. For a fixed $\beta$, the expectation term in 15) with the MMSE denoiser $\eta_{t, \beta}\left(\hat{\boldsymbol{x}}_{t, \beta}\right)=$ $\mathbb{E}\left[\boldsymbol{X}_{\beta} \mid \hat{\boldsymbol{X}}_{t, \beta}=\hat{\boldsymbol{x}}_{t, \beta}\right]$ can be expressed as [18]

$$
\begin{aligned}
& \mathbb{E}_{\hat{\boldsymbol{X}}_{t, \beta} \boldsymbol{X}_{\beta}}\left[\left(\eta_{t, \beta}\left(\hat{\boldsymbol{X}}_{t, \beta}\right)-\boldsymbol{X}_{\beta}\right)\left(\eta_{t, \beta}\left(\hat{\boldsymbol{X}}_{t, \beta}\right)-\boldsymbol{X}_{\beta}\right)^{H}\right] \\
& =\mathbb{E}_{\hat{\boldsymbol{X}}_{t, \beta}} \mathbb{E}_{\boldsymbol{X}_{\beta} \mid \hat{\boldsymbol{X}}_{t, \beta}=\hat{\boldsymbol{x}}_{t, \beta}}\left[\left(\mathbb{E}\left[\boldsymbol{X}_{\beta} \mid \hat{\boldsymbol{X}}_{t, \beta}=\hat{\boldsymbol{x}}_{t, \beta}\right]-\boldsymbol{X}_{\beta}\right)\right. \\
& \left.\left(\mathbb{E}\left[\boldsymbol{X}_{\beta} \mid \hat{\boldsymbol{X}}_{t, \beta}=\hat{\boldsymbol{x}}_{t, \beta}\right]-\boldsymbol{X}_{\beta}\right)^{H}\right] \\
& =\mathbb{E}_{\hat{\boldsymbol{X}}_{t, \beta}} \mathbb{E}\left[\boldsymbol{X}_{\beta} \boldsymbol{X}_{\beta}^{H} \mid \hat{\boldsymbol{X}}_{t, \beta}=\hat{\boldsymbol{x}}_{t, \beta}\right] \\
& -\mathbb{E}_{\hat{\boldsymbol{X}}_{t, \beta}} \mathbb{E}\left[\boldsymbol{X}_{\beta} \mid \hat{\boldsymbol{X}}_{t, \beta}=\hat{\boldsymbol{x}}_{t, \beta}\right]\left(\mathbb{E}\left[\boldsymbol{X}_{\beta} \mid \hat{\boldsymbol{X}}_{t, \beta}=\hat{\boldsymbol{x}}_{t, \beta}\right]\right)^{H} \\
& =\mathbb{E}_{\hat{\boldsymbol{X}}_{t, \beta}}\left[\phi_{t, \beta}\left(\beta \boldsymbol{I}-\beta^{2}\left(\beta \boldsymbol{I}+\boldsymbol{\Sigma}_{t}\right)^{-1}\right)+\right. \\
& \left.\phi_{t, \beta}\left(1-\phi_{t, \beta}\right) \beta^{2}\left(\beta \boldsymbol{I}+\boldsymbol{\Sigma}_{t}\right)^{-1} \hat{\boldsymbol{x}}_{t, \beta} \hat{\boldsymbol{x}}_{t, \beta}^{H}\left(\beta \boldsymbol{I}+\boldsymbol{\Sigma}_{t}\right)^{-1}\right],
\end{aligned}
$$

where the last equality is due to Lemma 1 and $\phi_{t, \beta}$ is used to denote $\phi\left(\hat{\boldsymbol{x}}_{t, \beta}\right)$.

According to 64], we have

$$
\phi_{t, \beta} p_{\hat{\boldsymbol{x}}_{t, \beta}}=\frac{\epsilon \exp \left(-\hat{\boldsymbol{x}}_{t, \beta}^{H}\left(\beta \boldsymbol{I}+\boldsymbol{\Sigma}_{t}\right)^{-1} \hat{\boldsymbol{x}}_{t, \beta}\right)}{\pi^{M}\left|\beta \boldsymbol{I}+\boldsymbol{\Sigma}_{t}\right|} .
$$

As a result, we have $\mathbb{E}_{\hat{\boldsymbol{X}}_{t, \beta}}\left[\phi_{t, \beta}\right]=\epsilon$. It then follows that

$$
\begin{aligned}
\mathbb{E}_{\hat{\boldsymbol{X}}_{t, \beta}}\left[\phi_{t, \beta}\left(\beta \boldsymbol{I}-\beta^{2}\left(\beta \boldsymbol{I}+\boldsymbol{\Sigma}_{t}\right)^{-1}\right)\right] & \\
= & \epsilon\left(\beta \boldsymbol{I}-\beta^{2}\left(\beta \boldsymbol{I}+\boldsymbol{\Sigma}_{t}\right)^{-1}\right) .
\end{aligned}
$$

By substituting 677 into 65], the expected MSE for estimating $\boldsymbol{X}_{\beta}$ given any $\beta$ after $t$ iterations is

$$
\begin{gathered}
\mathbb{E}_{\hat{\boldsymbol{X}}_{t, \beta}}\left[\left(\eta_{t, \beta}\left(\hat{\boldsymbol{X}}_{t, \beta}\right)-\boldsymbol{X}_{\beta}\right)\left(\eta_{t, \beta}\left(\hat{\boldsymbol{X}}_{t, \beta}\right)-\boldsymbol{X}_{\beta}\right)^{H}\right] \\
=\epsilon\left(\beta \boldsymbol{I}-\beta^{2}\left(\beta \boldsymbol{I}+\boldsymbol{\Sigma}_{t}\right)^{-1}\right)+\mathbb{E}_{\hat{\boldsymbol{X}}_{t, \beta}}\left[\phi_{t, \beta}\left(1-\phi_{t, \beta}\right) \beta^{2}\right. \\
\left.\left(\beta \boldsymbol{I}+\boldsymbol{\Sigma}_{t}\right)^{-1} \hat{\boldsymbol{X}}_{t, \beta} \hat{\boldsymbol{X}}_{t, \beta}^{H}\left(\beta \boldsymbol{I}+\boldsymbol{\Sigma}_{t}\right)^{-1}\right] .
\end{gathered}
$$

Note here $\phi_{t, \beta}$ is also a random variable depending on $\hat{\boldsymbol{X}}_{t, \beta}$ as in (19).

Based on the MSE shown in 688, in the following we show that $\boldsymbol{\Sigma}_{t}$ stays as a diagonal matrix with identical diagonal entries after each iteration of the AMP algorithm. 
First, it can be easily seen that for the initial state given in (16), $\boldsymbol{\Sigma}_{t}$ is indeed a diagonal matrix with identical diagonal elements, i.e., $\boldsymbol{\Sigma}_{0}=\tau_{0}^{2} \boldsymbol{I}$, with

$$
\tau_{0}^{2}=\frac{\sigma^{2}}{\xi}+\omega \epsilon \mathbb{E}[\beta] .
$$

Next, suppose that $\boldsymbol{\Sigma}_{t}=\tau_{t}^{2} \boldsymbol{I}$, given $t \geq 0$. We show in the following that $\boldsymbol{\Sigma}_{t+1}$ must be a diagonal matrix with identical diagonal elements, i.e., $\boldsymbol{\Sigma}_{t+1}=\tau_{t+1}^{2} \boldsymbol{I}$. Define

$$
\begin{aligned}
\boldsymbol{D} & =\mathbb{E}\left[\phi_{t, \beta}\left(1-\phi_{t, \beta}\right) \beta^{2}\left(\beta \boldsymbol{I}+\boldsymbol{\Sigma}_{t}\right)^{-1} \hat{\boldsymbol{X}}_{t, \beta} \hat{\boldsymbol{X}}_{t, \beta}^{H}\right. \\
& \left.\left(\beta \boldsymbol{I}+\boldsymbol{\Sigma}_{t}\right)^{-1}\right] \\
= & \mathbb{E}\left[\phi_{t, \beta}\left(1-\phi_{t, \beta}\right) \frac{\beta^{2}}{\left(\beta+\tau_{t}^{2}\right)^{2}} \hat{\boldsymbol{X}}_{t, \beta} \hat{\boldsymbol{X}}_{t, \beta}^{H}\right],
\end{aligned}
$$

where the distribution of $\hat{\boldsymbol{X}}_{t, \beta}$ given in (63) reduces to

$$
p_{\hat{\boldsymbol{x}}_{t, \beta}}=\frac{\epsilon \exp \left(-\frac{\hat{\boldsymbol{x}}_{t, \beta}^{H} \hat{\boldsymbol{x}}_{t, \beta}}{\beta+\tau_{t}^{2}}\right)}{\pi^{M}\left(\beta+\tau_{t}^{2}\right)^{M}}+\frac{(1-\epsilon) \exp \left(-\frac{\hat{\boldsymbol{x}}_{t, \beta}^{H} \hat{\boldsymbol{x}}_{t, \beta}}{\tau_{t}^{2}}\right)}{\pi^{M}\left(\tau_{t}^{2}\right)^{M}},
$$

and the random variable $\phi_{t, \beta}$ given in (19) reduces to

$$
\phi_{t, \beta}=\frac{1}{1+\frac{1-\epsilon}{\epsilon} \exp \left(-\left(\frac{1}{\tau_{t}^{2}}-\frac{1}{\beta+\tau_{t}^{2}}\right) \hat{\boldsymbol{x}}_{t, \beta}^{H} \hat{\boldsymbol{x}}_{t, \beta}\right)\left(1+\frac{\beta}{\tau_{t}^{2}}\right)^{M}} .
$$

For any $1 \leq i, j \leq M$, define $\boldsymbol{D}(i, j)$ as the element in the $i$ th row and $j$ th column of $\boldsymbol{D}$, and $\hat{\boldsymbol{X}}_{t, \beta}(i)$ as the $i$ th element of $\hat{\boldsymbol{X}}_{t, \beta}$. For the non-diagonal elements of $\boldsymbol{D}$, we have

$$
\begin{aligned}
& \boldsymbol{D}(i, j) \\
& =\mathbb{E}\left[\phi_{t, \beta}\left(1-\phi_{t, \beta}\right) \frac{\beta^{2}}{\left(\beta+\tau_{t}^{2}\right)^{2}} \hat{\boldsymbol{X}}_{t, \beta}(i) \hat{\boldsymbol{X}}_{t, \beta}^{H}(j)\right] \\
& =\int \phi_{t, \beta}\left(1-\phi_{t, \beta}\right) \frac{\beta^{2}}{\left(\beta+\tau_{t}^{2}\right)^{2}} \hat{\boldsymbol{x}}_{t, \beta}(i) \hat{\boldsymbol{x}}_{t, \beta}^{H}(j) p_{\hat{\boldsymbol{x}}_{t, \beta}} d \hat{\boldsymbol{x}}_{t, \beta},
\end{aligned}
$$

where $\hat{\boldsymbol{x}}_{t, \beta}(i)$ is the $i$ th element of $\hat{\boldsymbol{x}}_{t, \beta}$. The key observation is that $\phi_{t, \beta}$ as expressed in (72) and $p_{\hat{\boldsymbol{x}}_{t, \beta}}$ as expressed in (71) involves only square terms $\left|\hat{\boldsymbol{x}}_{t, \beta}(i)\right|^{2}$ so are both even functions, while $\hat{\boldsymbol{x}}_{t, \beta}(i) \hat{\boldsymbol{x}}_{t, \beta}^{H}(j)$ is an odd function for $i \neq j$, so the overall integral is zero. Hence $\boldsymbol{D}(i, j)=0$ for $i \neq j$, i.e., $\boldsymbol{D}$ is diagonal.

Next, consider the diagonal terms of $\boldsymbol{D}$

$$
\boldsymbol{D}(i, i)=\mathbb{E}\left[\phi_{t, \beta}\left(1-\phi_{t, \beta}\right) \frac{\beta^{2}}{\left(\beta+\tau_{t}^{2}\right)^{2}} \hat{\boldsymbol{X}}_{t, \beta}(i) \hat{\boldsymbol{X}}_{t, \beta}^{H}(i)\right] .
$$

It can be observed from (71) and (72) that all the elements of $\hat{\boldsymbol{X}}_{t, \beta}$ contribute equally to $p_{\hat{\boldsymbol{x}}_{t, \beta}}$ and $\phi_{t, \beta}$. Moreover, all the elements of $\hat{\boldsymbol{X}}_{t, \beta}$ have an identical distribution. As a result, we have $\boldsymbol{D}(i, i)=\boldsymbol{D}(j, j)=\vartheta_{t, \beta}\left(\tau_{t}^{2}\right), 1 \leq i, j \leq M$, i.e., $\boldsymbol{D}=\vartheta_{t, \beta}\left(\tau_{t}^{2}\right) \boldsymbol{I}$. To compute $\vartheta_{t, \beta}\left(\tau_{t}^{2}\right)$, we observe that

$$
\vartheta_{t, \beta}\left(\tau_{t}^{2}\right)=\frac{\operatorname{tr}(\boldsymbol{D})}{M}=\frac{\mathbb{E}\left[\phi_{t, \beta}\left(1-\phi_{t, \beta}\right) \frac{\beta^{2}}{\left(\beta+\tau_{t}\right)^{2}} \hat{\boldsymbol{X}}_{t, \beta}^{H} \hat{\boldsymbol{X}}_{t, \beta}\right]}{M} \text {. }
$$

This proves 29).

Finally, putting the above expression for $D$ back to 68 and recognizing that if $\boldsymbol{\Sigma}_{t}=\tau_{t}^{2} \boldsymbol{I}$, then

$$
\epsilon\left(\beta \boldsymbol{I}-\beta_{n}^{2}\left(\beta_{n} \boldsymbol{I}+\boldsymbol{\Sigma}_{t}\right)^{-1}\right)=\epsilon\left(\frac{\beta \tau_{t}^{2}}{\beta+\tau_{t}^{2}} \boldsymbol{I}\right),
$$

so by the state evolution (15), this implies that if $\boldsymbol{\Sigma}_{t}=\tau_{t}^{2} \boldsymbol{I}$, we have

$$
\boldsymbol{\Sigma}_{t+1}=\frac{\sigma^{2}}{\xi} \boldsymbol{I}+\omega\left(\epsilon \mathbb{E}\left[\frac{\beta \tau_{t}^{2}}{\beta+\tau_{t}^{2}}\right] \boldsymbol{I}+\mathbb{E}\left[\vartheta_{t, \beta}\left(\tau_{t}^{2}\right)\right] \boldsymbol{I}\right),
$$

which is also a diagonal matrix with identical diagonal elements, i.e., $\boldsymbol{\Sigma}_{t+1}=\tau_{t+1}^{2} \boldsymbol{I}$. The scalar form of the denoiser (24)-(26) and the scalar state evolution (28) then follow immeidately. Theorem 1 is thus proved.

\section{Proof of Theorem 2}

According to the state evolution equivalent signal model (23) in Theorem 11 when $\alpha_{n}=0$, the entries of $\left(\boldsymbol{R}^{t}\right)^{H} \boldsymbol{a}_{n}+$ $\boldsymbol{x}_{n}^{t}$ are i.i.d. Gaussian distributed with variance $\tau_{t}^{2}$. When $\alpha_{n}=1$, the entries of $\left(\boldsymbol{R}^{t}\right)^{H} \boldsymbol{a}_{n}+\boldsymbol{x}_{n}^{t}$ are i.i.d. Gaussian distributed with variance $\beta_{n}+\tau_{t}^{2}$. Since $\left(\boldsymbol{R}^{t}\right)^{H} \boldsymbol{a}_{n}+\boldsymbol{x}_{n}^{t}$ given $\alpha_{n}$ is a complex Gaussian random vector with i.i.d. real and imaginary components, the random variables $\left(\left(\boldsymbol{R}^{t}\right)^{H} \boldsymbol{a}_{n}+\boldsymbol{x}_{n}^{t}\right)^{H}\left(\left(\boldsymbol{R}^{t}\right)^{H} \boldsymbol{a}_{n}+\boldsymbol{x}_{n}^{t}\right) /\left(2 \tau_{t}^{2}\right)$ given $\alpha_{n}=0$ and $\left(\left(\boldsymbol{R}^{t}\right)^{H} \boldsymbol{a}_{n}+\boldsymbol{x}_{n}^{t}\right)^{H}\left(\left(\boldsymbol{R}^{t}\right)^{H} \boldsymbol{a}_{n}+\boldsymbol{x}_{n}^{t}\right) /\left(2\left(\beta_{n}+\tau_{t}^{2}\right)\right)$ given $\alpha_{n}=1$ thus follow $\chi^{2}$ distribution with $2 M$ DoF.

Let $X \sim \chi_{2 M}$ be a random variable that follows $\chi^{2}$ distribution with $2 M$ DoF. It is well known that its cumulative distribution function follows $\operatorname{Pr}(X \leq$ $x)=\frac{\Gamma\left(M, \frac{x}{2}\right)}{\Gamma(M)}$. The proposed detector (30) compares $\left(\left(\boldsymbol{R}^{t}\right)^{H} \boldsymbol{a}_{n}+\boldsymbol{x}_{n}^{t}\right)^{H}\left(\left(\boldsymbol{R}^{t}\right)^{H} \boldsymbol{a}_{n}+\boldsymbol{x}_{n}^{t}\right)$ with the threshold $M \log \left(1+\frac{\beta_{n}}{\tau_{t}^{2}}\right) /\left(\frac{1}{\tau_{t}^{2}}-\frac{1}{\tau_{t}^{2}+\beta_{n}}\right)$. By the definition of missed detection and false alarm probabilities (32) and (33), it thus follows that

$$
\begin{aligned}
P_{t, n}^{\mathrm{MD}}(M) & =\operatorname{Pr}\left(X \leq \frac{2 M \log \left(1+\frac{\beta_{n}}{\tau_{t}^{2}}\right)}{\left(\beta_{n}+\tau_{t}^{2}\right)\left(\frac{1}{\tau_{t}^{2}}-\frac{1}{\beta_{n}+\tau_{t}^{2}}\right)}\right) \\
& =\frac{\Gamma\left(M, b_{t, n} M\right)}{\Gamma(M)},
\end{aligned}
$$

and

$$
\begin{aligned}
P_{t, n}^{\mathrm{FA}}(M) & =\operatorname{Pr}\left(X \geq \frac{2 M \log \left(1+\frac{\beta_{n}}{\tau_{t}^{2}}\right)}{\tau_{t}^{2}\left(\frac{1}{\tau_{t}^{2}}-\frac{1}{\beta_{n}+\tau_{t}^{2}}\right)}\right) \\
& =1-\frac{\underline{\Gamma}\left(M, c_{t, n} M\right)}{\Gamma(M)}=\frac{\bar{\Gamma}\left(M, c_{t, n} M\right)}{\Gamma(M)},
\end{aligned}
$$


where

$$
\begin{aligned}
& b_{t, n}=\frac{\log \left(1+\frac{\beta_{n}}{\tau_{t}^{2}}\right)}{\left(\beta_{n}+\tau_{t}^{2}\right)\left(\frac{1}{\tau_{t}^{2}}-\frac{1}{\beta_{n}+\tau_{t}^{2}}\right)}=\frac{\tau_{t}^{2}}{\beta_{n}} \log \left(1+\frac{\beta_{n}}{\tau_{t}^{2}}\right), \\
& c_{t, n}=\frac{\log \left(1+\frac{\beta_{n}}{\tau_{t}^{2}}\right)}{\tau_{t}^{2}\left(\frac{1}{\tau_{t}^{2}}-\frac{1}{\beta_{n}+\tau_{t}^{2}}\right)}=\left(\frac{\beta_{n}+\tau_{t}^{2}}{\beta_{n}}\right) \log \left(1+\frac{\beta_{n}}{\tau_{t}^{2}}\right) .
\end{aligned}
$$

Theorem 2 is thus proved.

\section{Proof of Theorem 3}

Based on the vector AMP state evolution, the estimated channel and the corresponding channel estimation error, assuming that device $k$ is active, i.e., $\alpha_{k}=1$, is statistically equivalent to applying the denoiser (24) to the equivalent signal model (23) as shown below:

$$
\begin{aligned}
& \hat{\boldsymbol{h}}_{k}=\phi_{t, k}\left(\frac{\beta_{k}}{\beta_{k}+\tau_{\infty}^{2}}\right)\left(\boldsymbol{h}_{k}+\tau_{t} \boldsymbol{v}_{k}\right), \\
& \Delta \hat{\boldsymbol{h}}_{k}=\boldsymbol{h}_{k}-\hat{\boldsymbol{h}}_{k}, \quad \forall k \in \mathcal{K} .
\end{aligned}
$$

The main conclusion of Theorem 3 is that for any active user $k$, given any $M, \operatorname{Cov}\left(\hat{\boldsymbol{h}}_{k}, \hat{\boldsymbol{h}}_{k}\right)$ is a diagonal matrix with identical diagonal entries. The proof of this fact uses the same technique as used in Appendix B for proving Theorem 1, and so is not repeated here. Similarly, it can also be shown that $\operatorname{Cov}\left(\Delta \hat{\boldsymbol{h}}_{k}, \Delta \hat{\boldsymbol{h}}_{k}\right)$ is a diagonal matrix with identical diagonal entries. The expressions for the diagonal terms $v_{t, k}(M)$ and $\Delta v_{t, k}(M)$ (40)-(41) follow directly from (83)-884).

\section{E. Proof of Theorem 4}

First we show that assuming $\beta_{n}$ is bounded below, i.e., $\beta_{n}>\beta_{\text {min }}, \forall n$, we have $b_{t, n} \leq 1-\varepsilon_{t, n}^{(1)}, c_{t, n} \geq 1+\varepsilon_{t, n}^{(2)}$, $\nu_{t, n} \leq-\varepsilon_{t, n}^{(3)}, \varsigma_{t, n} \geq \varepsilon_{t, n}^{(4)}$, for some positive constants $\varepsilon_{t, n}^{(1)}, \varepsilon_{t, n}^{(2)}, \varepsilon_{t, n}^{(3)}$, and $\varepsilon_{t, n}^{(4)}$ that are independent of $M$. This is because it can be easily checked based on the state evolution equation (28) that $\tau_{t}^{2}$ is always bounded from above. So, $\beta_{n} / \tau_{t}^{2}$ is always lower bounded by some positive constant indepedent of $M$. Now, combining with the fact that $a>\log (1+a)>\frac{a}{1+a}$ for all $a>0$ with the first inequality becoming equality if and only if $a=1$, we can see that $b_{t, n}, c_{t, n}$, as in (36)-(37), and $\nu_{t, n}, \varsigma_{t, n}$ as in (45)-(46) are all bounded away from 1 and 0 , respectively, as required.

Next, we study the asymptotic probabilities of missed detection and false alarm. According to [29], if $b_{t, n} \leq 1-\varepsilon_{t, n}^{(1)}$ for some positive constant $\varepsilon_{t, n}^{(1)}$, then

$$
\begin{array}{r}
\frac{\underline{\Gamma}\left(M, b_{t, n} M\right)}{\Gamma(M)}=\frac{1}{2} \operatorname{efrc}\left(-\nu_{t, n} \sqrt{\frac{M}{2}}\right)-\frac{\exp \left(-\frac{1}{2} M \nu_{t, n}^{2}\right)}{\sqrt{2 \pi M}} \\
\left(\frac{1}{b_{t, n}-1}-\frac{1}{\nu_{t, n}}\right)-o\left(\frac{\exp (-M)}{\sqrt{M}}\right),
\end{array}
$$

where $\operatorname{efrc}(\cdot)$ is the complementary error function. Similarly, if $c_{t, n} \geq 1+\varepsilon_{t, n}^{(2)}$ for some positive constant $\varepsilon_{t, n}^{(2)}$, then

$$
\begin{gathered}
\frac{\bar{\Gamma}\left(M, c_{t, n} M\right)}{\Gamma(M)}=\frac{1}{2} \operatorname{efrc}\left(\varsigma_{t, n} \sqrt{\frac{M}{2}}\right)+\frac{\exp \left(-\frac{1}{2} M \varsigma_{t, n}^{2}\right)}{\sqrt{2 \pi M}} \\
\left(\frac{1}{c_{t, n}-1}-\frac{1}{\varsigma_{t, n}}\right)+o\left(\frac{\exp (-M)}{\sqrt{M}}\right) \cdot
\end{gathered}
$$

Moreover, it is known that

$$
\operatorname{efrc}(x)=\frac{\exp \left(-x^{2}\right)}{\sqrt{\pi} x}\left(1+o\left(\frac{1}{x^{2}}\right)\right) .
$$

Then, the missed detection probability given in 34 is

$$
\begin{aligned}
P_{t, n}^{\mathrm{MD}}(M)= & \frac{1}{2} \frac{\Gamma\left(M, b_{t, n} M\right)}{\Gamma(M)} \\
= & \frac{1}{2} \frac{\exp \left(-\frac{1}{2} M \nu_{t, n}^{2}\right)}{-\nu_{t, n} \sqrt{\pi M / 2}}\left(1+o\left(\frac{1}{M}\right)\right) \\
& -\frac{1}{2} \frac{\exp \left(-\frac{1}{2} M \nu_{t, n}^{2}\right)}{\sqrt{2 \pi M}}\left(\frac{1}{b_{t, n}-1}-\frac{1}{\nu_{t, n}}\right) \\
& -o\left(\frac{\exp (-M)}{\sqrt{M}}\right) \\
= & -\frac{\exp \left(-\frac{1}{2} M \nu_{t, n}^{2}\right)}{2 \sqrt{2 \pi M}}\left(\frac{1}{b_{t, n}-1}+\frac{1}{\nu_{t, n}}\right) \\
& +o\left(\frac{\exp (-M)}{\sqrt{M}}\right),
\end{aligned}
$$

where (88) is obtained by applying (87) since $\nu_{t, n}<0$ with $b_{t, n}<1$. and the false alarm probability given in (35) is

$$
\begin{aligned}
P_{t, n}^{\mathrm{FA}}(M)= & \frac{1}{2} \frac{\bar{\Gamma}\left(M, b_{t, n} M\right)}{\Gamma(M)} \\
= & \frac{1}{2} \frac{\exp \left(-\frac{1}{2} M \varsigma_{t, n}^{2}\right)}{\varsigma_{t, n} \sqrt{\pi M / 2}}\left(1+o\left(\frac{1}{M}\right)\right) \\
& +\frac{1}{2} \frac{\exp \left(-\frac{1}{2} M \varsigma_{t, n}^{2}\right)}{\sqrt{2 \pi M}}\left(\frac{1}{c_{t, n}-1}-\frac{1}{\varsigma_{t, n}}\right) \\
& +o\left(\frac{\exp (-M)}{\sqrt{M}}\right) \\
= & \frac{\exp \left(-\frac{1}{2} M \varsigma_{t, n}^{2}\right)}{2 \sqrt{2 \pi M}}\left(\frac{1}{c_{t, n}-1}+\frac{1}{\varsigma_{t, n}}\right) \\
& +o\left(\frac{\exp (-M)}{\sqrt{M}}\right) .
\end{aligned}
$$

Theorem 4 is thus proved.

\section{F. Proof of Theorem 5}

Examining the functional form of $\vartheta_{t, \beta}\left(\tau_{t}^{2}\right)$ :

$\vartheta_{t, \beta}\left(\tau_{t}^{2}\right)=\mathbb{E}_{\hat{\boldsymbol{X}}_{t, \beta}}\left[\phi_{t, \beta}\left(1-\phi_{t, \beta}\right) \frac{\beta^{2}}{\left(\beta+\tau_{t}^{2}\right)^{2}} \frac{\hat{\boldsymbol{X}}_{t, \beta}^{H} \hat{\boldsymbol{X}}_{t, \beta}}{M}\right]$,

we see that as $0 \leq \phi_{t, \beta} \leq 1$ and by the law of large numbers, the term inside expectation is bounded from above by a 
constant independent of $M$. Thus, we can apply dominated convergence theorem for taking limit as $M \rightarrow \infty$, i.e.,

$$
\begin{array}{r}
\lim _{M \rightarrow \infty} \vartheta_{t, \beta}\left(\tau_{t}^{2}\right)=\mathbb{E}_{\hat{\boldsymbol{X}}_{t, \beta}}\left[\lim _{M \rightarrow \infty} \phi_{t, \beta}\left(1-\phi_{t, \beta}\right)\right. \\
\left.\frac{\beta^{2}}{\left(\beta+\tau_{t}^{2}\right)^{2}} \frac{\hat{\boldsymbol{X}}_{t, \beta}^{H} \hat{\boldsymbol{X}}_{t, \beta}}{M}\right] .
\end{array}
$$

But $\lim _{M \rightarrow \infty} \phi_{t, n}$ is either 0 or 1 , according to (48) as consequence of Theorem 4. It thus follows that

$$
\lim _{M \rightarrow \infty} \vartheta_{t, \beta}\left(\tau_{t}^{2}\right)=0 .
$$

\section{G. Proof of Theorem 6}

Assuming fixed $\tau_{t}^{2}$ as given by the simplified state evolution (50) in the massive MIMO regime in the limit as $M$ goes to infinity, we evaluate $\lim _{M \rightarrow \infty} v_{k}(M)$ and $\lim _{M} \Delta v_{k}(M)$ where $v_{k}(M)$ and $\Delta v_{k}(M)$ are as given in (40) and (41), respectively.

It is easy to check that since $0 \leq \phi_{t, k}^{2} \leq 1$ according to (19), it follows that

$$
\begin{aligned}
v_{k}(M) & \leq \mathbb{E}\left[\frac{\beta_{k}^{2}}{\left(\beta_{k}+\tau_{t}^{2}\right)^{2}} \frac{\left(\boldsymbol{h}_{k}+\tau_{t} \boldsymbol{v}_{k}\right)^{H}\left(\boldsymbol{h}_{k}+\tau_{t} \boldsymbol{v}_{k}\right)}{M}\right] \\
& =\frac{\beta_{k}^{2}}{\beta_{k}+\tau_{t}^{2}} .
\end{aligned}
$$

So $v_{k}(M)$ is upper bounded by a finite constant indepedent of $M$, we can then apply the dominated convergence theorem to compute

$$
\begin{aligned}
& \lim _{M \rightarrow t} v_{k}(M) \\
& =\mathbb{E}\left[\lim _{M \rightarrow t} \frac{\phi_{t, k}^{2} \beta_{k}^{2}}{\left(\beta_{k}+\tau_{t}^{2}\right)^{2}} \frac{\left(\boldsymbol{h}_{k}+\tau_{t} \boldsymbol{v}_{k}\right)^{H}\left(\boldsymbol{h}_{k}+\tau_{t} \boldsymbol{v}_{k}\right)}{M}\right] \\
& =\mathbb{E}\left[\frac{\beta_{k}^{2}}{\left(\beta_{k}+\tau_{t}^{2}\right)^{2}} \lim _{M \rightarrow t} \frac{\left(\boldsymbol{h}_{k}+\tau_{t} \boldsymbol{v}_{k}\right)^{H}\left(\boldsymbol{h}_{k}+\tau_{t} \boldsymbol{v}_{k}\right)}{M}\right] \\
& =\frac{\beta_{k}^{2}}{\beta_{k}+\tau_{t}^{2}},
\end{aligned}
$$

where (96) is due to (48) as consequence of Theorem 4

Similarly, as $M \rightarrow \infty$, we can show that $\Delta v_{k}(M)$ converges to $\frac{\beta_{k} \tau_{\infty}^{2}}{\beta_{k}+\tau_{\infty}^{2}}$.

\section{REFERENCES}

[1] L. Liu and W. Yu, "Massive device connectivity with massive MIMO,' in Proc. IEEE Inter. Symp. Inf. Theory (ISIT), Jun. 2017.

[2] C. Bockelmann, N. Pratas, H. Nikopour, K. Au, T. Svensson, C. Stefanovic, P. Popovsk, and A. Dekorsy, "Massive machine-type communications in 5G: Physical and MAC-layer solutions," IEEE Commun. Mag., vol. 54, no. 9, pp. 59-65, Sep. 2016.

[3] W. Yu, "On the fundamental limits of massive connectivity," in Proc. Inf. Theory and Appl. (ITA) Workshop, Feb. 2017.

[4] D. L. Donoho, A. Maleki, and A. Montanari, "Message-passing algorithms for compressed sensing," Proc. Nat. Acad. Sci., vol. 106, no. 45, pp. 18914-18918, Nov. 2009.

[5] L. Liu and W. Yu, "Massive connectivity with massive MIMO-Part II: Achievable rate characterization," IEEE Trans. Signal Process., 2018, to be published.
[6] M. Hasan, E. Hossain, and D. Niyato, "Random access for machineto-machine communication in LTE-advanced networks: Issues and approaches,“ IEEE Commun. Mag., vol. 51, no. 6, pp. 86-93, Jun. 2011.

[7] N. K. Pratas, H. Thomsen, C. Stefanovic, and P. Popovski, "Codeexpanded random access for machine-type communications, " in Proc. IEEE Globecom Workshops, Dec. 2012, pp. 1681-1686.

[8] E. Björnson, E. de Carvalho, J. H. Sørensen, E. G. Larsson, and P. Popovski, "A random access protocol for pilot allocation in crowded massive MIMO systems, "IEEE Trans. Wireless Commun., vol. 16, no. 4, pp. 2220-2234, Apr. 2017..

[9] H. Zhu and G. B. Giannakis, "Exploiting sparse user activity in multiuser detection," IEEE Trans. Commun., vol. 59, no. 2, pp. 454465, Feb. 2011.

[10] H. F. Schepker and A. Dekorsy, "Compressive sensing multi-user detection with block-wise orthogonal least squares, " in Proc. IEEE Veh. Tech. Conf. (VTC Spring), May 2012, pp. 1-5.

[11] X. Xu, X. Rao, and V. K. N. Lau, "Active user detection and channel estimation in uplink CRAN systems," in Proc. IEEE Int. Conf. Commun. (ICC), Jun. 2015, pp. 2727-2732.

[12] G. Wunder, P. Jung, and M. Ramadan, "Compressive random access using a common overloaded control channel," in Proc. IEEE Int. Conf. Commun. (ICC) Workshops, Jun. 2015.

[13] G. Hannak, M. Mayer, A. Jung, G. Matz, and N. Goertz, "Joint channel estimation and activity detection for multiuser communication systems," in Proc. IEEE Int. Conf. Commun. (ICC) Workshops, Jun. 2015, pp. 2086-2091.

[14] H. F. Schepker, C. Bockelmann, and A. Dekorsy, "Exploiting sparsity in channel and data estimation for sporadic multi-user communication," in Inter. Symp. Wireless Commun. Sys. (ISWCS), Aug. 2013, pp. 1-5.

[15] G. Wunder, H. Boche, T. Strohmer, and P. Jung, "Sparse signal processing concepts for efficient 5G system design, “ IEEE Access, vol. 3, pp. 195-208, 2015.

[16] G. Wunder, P. Jung, and C. Wang, "Compressive random access for post-LTE systems," in Proc. IEEE Int. Conf. Commun. (ICC) Workshops, Jun. 2014.

[17] Z. Chen and W. Yu, "Massive device activity detection by approximate message passing," in Proc. IEEE Int. Conf. Acoustic, Speech, Signal Process. (ICASSP), Mar. 2017.

[18] Z. Chen, F. Sohrabi, and W. Yu, "Sparse activity detection for massive connectivity,“ IEEE Trans. Signal Process., vol. 66, no. 7, pp. 18901904, Apr. 2018

[19] M. Bayati and A. Montanari, "The dynamics of message passing on dense graphs, with applications to compressed sensing," IEEE Trans. Inf. Theory, vol. 57, no. 2, pp. 764-785, Feb. 2011.

[20] T. L. Marzetta, "Noncooperative cellular wireless with unlimited numbers of base station antennas," IEEE Trans. Wireless Commun., vol. 9, no. 11, pp. 3590-3660, Nov. 2010.

[21] H. Q. Ngo, E. G. Larsson, and T. L. Marzetta, "Energy and spectral efficiency of very large multiuser MIMO systems," IEEE Trans. Commun., vol. 61, no. 4, pp. 1436-1449, Apr. 2013.

[22] J. Hoydis, S. Brink, and M. Debbah, "Massive MIMO in the UL/DL of cellular networks: How many antennas do we needi " IEEE J. Sel. Areas Commun., vol. 31, no. 2, pp. 160-171, Feb. 2013.

[23] E. G. Larsson, F. Tufvesson, O. Edfors, and T. L. Marzetta, "Massive MIMO for next generation wireless systems," IEEE Commun. Mag., vol. 52, no. 2, pp. 186-195, Feb. 2014.

[24] S. Wagner, R. Couillet, M. Debbah, and D. T. M. Slock, "Large system analysis of linear precoding in correlated MISO broadcast channels under limited feedback," IEEE Trans. Inf. Theory, vol. 58, no. 7, pp. 4509-4537, Jul. 2012.

[25] X. Chen, T.-Y. Chen, and D. Guo, "Capacity of Gauassian manyaccess channels," IEEE Trans. Inf. Theory, vol. 63, no. 6, pp. 35163519, Jun. 2017.

[26] J. Kim, W. Chang, B. Jung, D. Baron, and J. C. Ye, "Belief propagation for joint sparse recovery, “ Feb. 2011, [Online] Available: http://arxiv.org/abs/1102.3289

[27] J. Ziniel and P. Schniter, "Efficient high-dimensional inference in the multiple measurement vector problem," IEEE Trans. Signal Process., vol. 61, no. 2, pp. 340-354, Jan. 2013.

[28] S. Rangan, "Generalized approximate message passing for estimation with random linear mixing," in Proc. IEEE Int. Symp. Inf. Theory (ISIT), Jul. 2011, pp. 2168-2172.

[29] W. Gautschi, "The incomplete gamma functions since Tricomi," Atti dei Convegni Linci, no. 1998, pp. 203-237, 2011 